The Astrophysical Journal, 249:481-503, 1981 October 15

() 1981. The American Astronomical Society. All rights reserved. Printed in U.S.A.

\title{
BOLOMETRIC LUMINOSITIES AND INFRARED PROPERTIES OF CARBON STARS IN THE MAGELLANIC CLOUDS AND THE GALAXY
}

\author{
JUDITH G. COHEN \\ Palomar Observatory, California Institute of Technology \\ JAY A. FrogeL ${ }^{1}$ \\ Cerro Tololo Inter-American Observatory ${ }^{2}$ \\ S. E. Persson \\ Mount Wilson and Las Campanas Observatories, Carnegie Institution of Washington \\ AND \\ JONATHAN H. ELIAS \\ Cerro Tololo Inter-American Observatory ${ }^{2}$ \\ Received 1980 December 1; accepted 1981 April 27
}

\begin{abstract}
Broad band $J, H, K$ photometry and narrow band $\mathrm{CO}$ and $\mathrm{H}_{2} \mathrm{O}$ indices have been obtained for 89 luminous red stars in the Large Magellanic Cloud (LMC) and 21 in the Small Magellanic Cloud (SMC), chosen largely from the sample of Blanco, McCarthy, and Blanco. Most are known to be carbon stars, and their infrared properties are compared with new observations of 33 galactic carbon stars. The bolometric luminosity distributions of an unbiased sample of Magellanic Cloud carbon stars are compared with those predicted from evolutionary calculations by Renzini and Voli for double shell burning stars undergoing He shell flashes. The observed and theoretical distributions disagree markedly: nearly all the observed stars have lower luminosities than even the faintest theoretical carbon star.

In addition, we find many fewer than expected high luminosity stars with initial mass greater than $3 M_{\odot}$. Possible explanations for this include a steep initial mass function for intermediate mass stars, a star formation rate significantly higher in the past than at present, or a neglected physical effect, such as underestimation of the importance of mass loss. Nevertheless, it appears that the hypothesis that He shell flashes lead to a dredge-up of carbon into the envelope, which results in a carbon star, can be maintained, if dredging occurs after fewer shell flashes than are predicted by presently available stellar evolutionary calculations.

The colors and indices of the late M giants in the LMC field are similar to those of late $\mathrm{M}$ giants in the Galaxy.

The narrow band infrared data are interpreted qualitatively in terms of the effects of molecular band absorption, which also strongly influences the infrared broad band colors of carbon stars. The small differences in the color-color relationships of the SMC and LMC samples are consistent with the differences in heavy metal abundance between the LMC, SMC, and Galaxy.
\end{abstract}

Subject headings: galaxies: Magellanic Clouds - galaxies: stellar content - luminosity function stars: abundances - stars: carbon - stars: luminosities

\section{INTRODUCTION}

The recent deep transmission grating prism survey at $\lambda=8100 \AA(=I)$ of selected fields in the Magellanic Clouds by Blanco, McCarthy, and Blanco (1980, hereafter BMB) has revealed the presence of large numbers of carbon stars. Although the presence of luminous C stars in the Magellanic Clouds had been known from

\footnotetext{
${ }^{1}$ Guest Investigator, Las Campanas Observatory.

${ }^{2}$ Cerro Tololo Inter-American Observatory is supported by the
} National Science Foundation under Contract No. AST 78-27879. earlier, large scale surveys (e.g., Westerlund 1960, 1964; Westerlund et al. 1978, hereafter WORC; Sanduleak and Philip 1977), the primary result from the BMB survey is how important the $\mathrm{C}$ stars are to the late-type giant population: the ratio of luminous $\mathrm{C}$ stars to luminous M stars later than M4 is 27 in the SMC and 2 in the LMC, compared with 0.003 in the nuclear bulge of our Galaxy (Blanco, Blanco, and McCarthy 1978). $\mathrm{BMB}$ suggested that the dramatic differences in $\mathrm{C}$ to $\mathrm{M}$ star ratios between the Milky Way, the Large Magellanic Cloud (LMC), and the Small Magellanic Cloud 
(SMC) are related to the metallicity differences between the three systems. It is by now well established that on the whole the SMC is deficient in heavy elements compared to the LMC, and th LMC is probably somewhat metal-poor compared to the solar neighborhood (Osmer 1976; Peimbert and Torres-Peimbert 1976; Dufour and Harlow 1977).

As well as providing unique new information on the stellar content of the Magellanic Clouds, the BMB survey is a milestone in research on the carbon star phenomenon itself. For the first time we have available a large, virtually complete sample of $\mathrm{C}$ stars at a known distance. Theoretical studies of $\mathrm{C}$ star formation and evolution (e.g., Iben and Truran 1978; Renzini and Voli 1981) make specific predictions regarding luminosities and luminosity functions, and these predictions can now be tested quantitatively. Sackmann, Smith, and Despain (1974) and Renzini and Voli have also begun to study the formation of $\mathrm{C}$ stars as a function of chemical abundance.

Since the late $\mathrm{C}$ and $\mathrm{M}$ stars in the Magellanic Clouds emit most of their energy longward of $1 \mu \mathrm{m}$, near-infrared observations are clearly desirable in determining their bolometric luminosities. We have therefore studied a sample of the BMB C and M stars in the LMC and SMC using broad and narrow band infrared photometry to measure $J, H$, and $K$ magnitudes and CO and $\mathrm{H}_{2} \mathrm{O}$ indices. Similar data have been obtained for a sample of the visually more luminous $C$ stars found in the WORC survey (cf. Richer, Olander, and Westerlund 1979). For comparison purposes, new infrared data have also been obtained for a representative sample of carbon stars in the Galaxy selected from the list of Walker (1979).

A second objective of this paper is to investigate how the infrared photometric properties of $\mathrm{C}$ stars depend on band absorption by carbon-bearing molecules. We will show that the effects of molecular blanketing and effective temperature upon the infrared colors and indices are inextricably entangled.

The outline of the paper is as follows: The selection of stars and observational techniques are discussed in $\S$ II. We then present the data, the basic correlations between infrared colors, magnitudes, and indices, and compare the various samples of $\mathrm{C}$ stars semiquantitatively in § III. In § IV bolometric luminosities are derived for the entire BMB sample. The resulting luminosity function is compared with theoretical predictions for stars undergoing shell flashes in the double shell burning evolutionary phase. The properties of the late $\mathrm{M}$ giants in the LMC field found by the BMB survey are also discussed in that section. In $\S \mathrm{V} \mathrm{a}$ physical basis for the correlations in infrared colors and indices based on line blanketing produced by molecules containing $\mathrm{C}$ atoms is proposed and is used to analyze the causes of the small but real differences between the infrared color-color relationships of LMC and SMC field C stars. Section VI gives a summary of the results. In the Appendix we explain how individual reddening values were obtained for the galactic $\mathrm{C}$ stars and how the various samples of Magellanic Cloud Stars were dereddened.

Some of the preliminary results of this paper were presented at the Erice Workshop on Physical Processes in Red Giants (Frogel et al. 1980).

\section{THE SELECTION AND OBSERVATION OF THE STARS}

Seventy-four $C$ and $M$ stars in four of the five areas of the BMB survey of the LMC and SMC were selected for observation in the infrared. Although we included stars whose properties cover the full range of color, magnitude, and spectral type in the survey, the actual distribution of these 74 stars is weighted toward the redder and brighter ones. These data were obtained primarily with the CTIO InSb system and f/30 chopping secondary on the $4 \mathrm{~m}$ telescope. Generally, a 5"'3 diameter diaphragm was employed to minimize crowding problems. The data are given in Tables 1-4.

Twenty-six of the $\mathrm{C}$ stars found in the WORC survey were observed in the infrared. These were selected to include essentially all of the stars with VRI photometry and optical spectra by Richer, Olander, and Westerlund (1979). Thus, whatever biases were present in their selection criteria are preserved in our sample. These data were obtained primarily with the Las Campanas InSb system on the $2.5 \mathrm{~m}$ du Pont telescope equipped with a re-imaging focal plane chopper. A 12."6 diameter diaphragm was used. These data are given in Table 5.

For both the BMB and the WORC stars, Quantex acquisition cameras were used to check the field of each star for other stars in the "signal" and "sky" beams. Nevertheless, we cannot rule out the possibility, particularly for the fainter objects, that in addition to the statistical uncertainties noted in Tables 1-5 the magnitudes are in error by up to about $5 \%$ because of contamination by neighboring stars. Such problems are expected to have a minimal effect, of course, on the colors and indices. As noted in the tables, six of these stars were measured on both the 2.5 and $4 \mathrm{~m}$ telescopes with no more than a few days separating the two observations. The mean dispersions in the magnitudes, colors, and indices are $\pm 0.02 \mathrm{mag}$ or less, consistent with the quoted uncertainties.

Finally, 33 galactic $\mathrm{C}$ stars, representative of a range in color and spectral type, were selected from the list of Walker (1979) and observed on the CTIO $0.9 \mathrm{~m}$ telescope with a rotating sector wheel chopper near the focal plane. These data are listed in Table 6.

All of the data in Tables 1-6 are referred to the system of standard stars given by Aaronson, Frogel, and Persson (1978) and by Frogel et al. (1978). Although 
TABLE 1

INFRARED OBSERVATIONS OF LMC “O” (OPTICAL CENTER) STARS ${ }^{a}$

\begin{tabular}{|c|c|c|c|c|c|c|c|}
\hline \multirow[b]{2}{*}{ STAR } & \multirow[b]{2}{*}{$\mathrm{Sp}$} & \multicolumn{5}{|c|}{ OBSERVED VALUES $^{\mathrm{b}}$} & \multirow[b]{2}{*}{ Notes } \\
\hline & & $K$ & $(J-K)$ & $(H-K)$ & $\mathrm{H}_{2} \mathrm{O}$ & $\mathrm{CO}$ & \\
\hline $2 \ldots$ & M6.0 & 11.93 & 1.15 & 0.38 & & & 3 \\
\hline $3 \ldots$ & M6.0 & 10.82 & 1.34 & 0.38 & 0.185 & +0.23 & 1 \\
\hline $7 \ldots$ & M6.5 & 10.97 & 1.28 & 0.35 & 0.17 & +0.24 & 4 \\
\hline $13 \ldots$ & C & 10.01 & 1.76 & 0.68 & 0.16 & -0.01 & 2 \\
\hline $16 \ldots$ & M7.0 & 11.38 & 1.14 & 0.36 & $0.40(4)$ & $+0.24(3)$ & 2 \\
\hline $17 \ldots$ & C & 10.45 & 1.67 & 0.61 & 0.155 & +0.035 & 2 \\
\hline $26 \ldots$ & C & 10.72 & 1.57 & 0.53 & 0.135 & +0.115 & 2 \\
\hline $33 \ldots$ & C & 10.02 & 1.90 & 0.73 & 0.205 & -0.04 & 2 \\
\hline $40 \ldots$ & $\mathrm{C}$ & 10.86 & 1.86 & 0.71 & 0.22 & -0.06 & 2 \\
\hline $42 \ldots$ & M7.0 & 10.78 & 1.23 & 0.32 & 0.175 & +0.22 & 1 \\
\hline $43 \ldots$ & C & 10.58 & 1.55 & 0.53 & 0.16 & +0.04 & 4 \\
\hline $47 \ldots$ & $\mathrm{C}$ & 10.78 & 1.23 & 0.33 & 0.09 & +0.19 & 4 \\
\hline $57 \ldots$ & M6.5 & 10.72 & 1.24 & 0.35 & 0.16 & +0.28 & 4 \\
\hline $75 \ldots$ & M8.0 & 10.89 & 1.23 & 0.36 & 0.345 & +0.27 & 3 \\
\hline $86 \ldots$ & $\mathrm{C}$ & 10.68 & $2.45(3)$ & 1.10 & 0.30 & -0.13 & 2 \\
\hline $97 \ldots$ & M7.5 & 11.09 & 1.25 & 0.36 & 0.245 & +0.25 & 3 \\
\hline $110 \ldots$ & $\mathrm{C}$ & 11.94 & 1.22 & 0.35 & $0.06(4)$ & $+0.105(4)$ & 3 \\
\hline $114 \ldots$ & $\mathrm{C}$ & 9.66 & 1.80 & 0.69 & 0.195 & -0.03 & 3 \\
\hline \multicolumn{2}{|c|}{ Corrections } & -0.03 & -0.07 & -0.02 & -0.005 & +0.005 & 5 \\
\hline
\end{tabular}

${ }^{a}$ The identification numbers and spectral types are from Blanco et al. 1980.

${ }^{b}$ The statistical uncertainties in the infrared magnitudes, colors, and indices are $\pm 0.02 \mathrm{mag}$ or less unless otherwise noted.

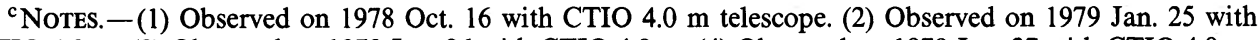
CTIO $4.0 \mathrm{~m}$. (3) Observed on $1979 \mathrm{Jan} .26$ with CTIO $4.0 \mathrm{~m}$. (4) Observed on $1979 \mathrm{Jan}$. 27 with CTIO $4.0 \mathrm{~m}$. (5) These are reddening corrections to be applied to the observed values. They are based on an $E(B-V)=0.12$ as discussed in the text.

most of the carbon stars are considerably redder than the reddest standard, unpublished observations of a number of extremely red stars indicate that the data in Tables 1-6 are in fact all on the standard system.

In this paper all magnitudes and colors were corrected for extinction and reddening following procedures described in the Appendix. These corrections were determined individually for each of the galactic carbon stars. Corrected values of magnitudes, colors, and indices are given in Table 6. Mean corrections for each of the BMB fields in the Magellanic Clouds were determined, and the values are indicated on the last line of Tables 1-4. Mean corrections for the LMC as a whole were used for the WORC stars and are given at the bottom of Table 5 .

\section{THE COLORS, MAGNITUDES, AND INDICES OF THE STARS}

In this section we present the data in graphical form and give the relations that exist between the observed quantities for each sample. We also point out the differences and similarities between the different samples of $C$ stars and between the $C$ stars and $M$ stars. We did not find any significant differences in the colors and indices for stars from the three fields within the LMC, consistent with the results of BMB. Henceforth, then, stars from the three LMC fields will be considered together.

\section{a) The Carbon Stars \\ i) Energy Distributions}

The LMC and SMC C and M stars are qualitatively similar to their counterparts in the Galaxy. Figures 1 and 2 show the $\left[(J-H)_{0},(H-K)_{0}\right]$-diagram for the galactic, LMC, and SMC stars. The $(J-H)_{0}$ and $(H-K)_{0}$ colors are highly correlated, in agreement with the results of Frogel and Hyland (1972) and Walker (1980). In the region $0.3 \leq(H-K)_{0} \leq 0.8$ a least squares fit to the data for the galactic $\mathrm{C}$ stars was determined by averaging the fits found by taking first one and then the other color as the independent variable. (The stars with $A_{V}<0.4$ were given double weight.) Since the points which exhibit the greatest scatter are those with the larger reddening values, and hence greater uncertainties, it is quite possible that there is very little intrinsic dispersion to this color-color relationship. The data of Frogel and Hyland are consistent with this interpretation. Of the four stars which are reddest in $(H-K)_{0}$, three are Mira variables, while the fourth is of unknown 
TABLE 2

INFRARED OBSERVATIONS OF LMC “BW” (BAR WEST) STARS ${ }^{a}$

\begin{tabular}{|c|c|c|c|c|c|c|c|}
\hline \multirow[b]{2}{*}{ STAR } & \multirow[b]{2}{*}{$\mathrm{Sp}$} & \multicolumn{5}{|c|}{ OBSERVED VALUes $^{\mathrm{b}}$} & \multirow[b]{2}{*}{ NOTES $^{\mathrm{c}}$} \\
\hline & & $K$ & $(J-K)$ & $(H-K)$ & $\mathrm{H}_{2} \mathrm{O}$ & $\mathrm{CO}$ & \\
\hline $1 \ldots$ & $\mathrm{C}$ & 10.80 & $1.75(3)$ & 0.61 & $0.15(3)$ & +0.04 & 1 \\
\hline $2 \ldots$ & M5.5 & 9.51 & 1.21 & 0.28 & 0.165 & +0.24 & 7 \\
\hline $3 \ldots$ & $\mathrm{C}$ & 10.26 & 1.53 & 0.52 & 0.13 & +0.08 & 3 \\
\hline $4 \ldots$ & M7.0 & 10.77 & 1.26 & 0.35 & $0.27(3)$ & $+0.26(3)$ & 7 \\
\hline $9 \ldots$ & $\mathrm{C}$ & 11.63 & $1.53(3)$ & 0.55 & $0.18(4)$ & $+0.03(3)$ & 1 \\
\hline $10 \ldots$ & M6.0 & 11.28 & $1.09(3)$ & 0.24 & 0.09 (3) & +0.19 & 1 \\
\hline $11 \ldots$ & C & 10.11 & $1.60(3)$ & 0.57 & $0.20(3)$ & +0.03 & 1 \\
\hline $19 \ldots$ & $\mathrm{C}$ & 11.20 & $1.17(3)$ & 0.27 & $0.085(3)$ & +0.26 & 1,5 \\
\hline $21 \ldots$ & $\mathrm{C}$ & 10.68 & $1.59(3)$ & 0.54 & $0.13(3)$ & +0.045 & 1 \\
\hline $24 \ldots$ & C & 10.41 & $1.60(3)$ & 0.55 & $0.13(3)$ & +0.045 & 1 \\
\hline $25 \ldots$ & $\mathrm{C}$ & 9.99 & 1.76 & 0.66 & 0.18 & -0.015 & 1,5 \\
\hline $34 \ldots$. & $\mathrm{C}$ & $10.43(3)$ & $1.90(3)$ & 0.78 & 0.22 & -0.045 & 4 \\
\hline $42 \ldots$ & $\mathrm{C}$ & 10.36 & $1.71(3)$ & 0.63 & $0.175(3)$ & 0.00 & 3 \\
\hline $44 \ldots$. & $\mathrm{C}$ & 10.73 & $1.64(3)$ & 0.59 & $0.155(3)$ & $+0.10(3)$ & 3 \\
\hline $48 \ldots$. & M6.0 & 10.22 & 1.25 & 0.29 & 0.095 & +0.215 & 7 \\
\hline $50 \ldots$ & M5.5 & 11.60 & $1.29(3)$ & 0.32 & $0.13(4)$ & $+0.24(3)$ & 3 \\
\hline $53 \ldots$ & C & $10.73(3)$ & $1.80(3)$ & 0.66 & 0.215 & 0.00 & 2 \\
\hline $54 \ldots$ & C & 9.71 & $1.44(3)$ & 0.47 & 0.14 & +0.15 & 3 \\
\hline $61 \ldots$ & M7.0 & $10.85(3)$ & $1.28(3)$ & 0.35 & 0.17 & +0.265 & 4 \\
\hline $68 \ldots$ & M7.5 & 10.51 & $1.23(3)$ & 0.36 & $0.22(3)$ & $+0.27(3)$ & 2,6 \\
\hline $78 \ldots$ & M7.5 & $10.17(3)$ & $1.19(3)$ & 0.37 & 0.26 & +0.315 & 4 \\
\hline $83 \ldots$ & M6.5 & $11.19(3)$ & $1.18(3)$ & 0.28 & $0.07(3)$ & +0.245 & 2 \\
\hline $85 \ldots$ & C & 9.78 & $1.89(3)$ & 0.73 & 0.205 & -0.01 & 3 \\
\hline $104 \ldots$ & C & 10.72 & 1.50 & 0.47 & $0.155(3)$ & +0.07 & 2 \\
\hline $105 \ldots$ & C & 10.22 & 1.76 & 0.66 & 0.18 & -0.02 & 2,5 \\
\hline $108 \ldots$ & $\mathrm{C}$ & $10.94(3)$ & $1.61(3)$ & 0.57 & $0.17(3)$ & $+0.04(3)$ & 2 \\
\hline \multicolumn{2}{|c|}{ Corrections } & -0.05 & -0.09 & -0.04 & -0.01 & +0.005 & 8,9 \\
\hline
\end{tabular}

${ }^{a}$ The identification numbers and spectral types are from Blanco et al. 1980.

${ }^{b}$ The combination of statistical and photometric uncertainties do not exceed $\pm 0.02 \mathrm{mag}$ in the colors, magnitudes, and indices unless otherwise noted.

${ }^{\text {c}}$ Notes. - (1) Observed on 1978 Dec. 16 with the CTIO $4 \mathrm{~m}$ telescope. (2) Observed on 1978 Dec. 17 with the CTIO $4 \mathrm{~m}$. (3) Observed on 1978 Dec. 18 with the CTIO $4 \mathrm{~m}$. (4) Observed on $1978 \mathrm{Dec} 19$ with the CTIO $4 \mathrm{~m}$. (5) Also observed with Las Campanas $2.5 \mathrm{~m}$ on $1978 \mathrm{Dec}$. 13. (6) Also observed with Las Campanas $2.5 \mathrm{~m}$ on 1978 Dec. 16. (7) Only observed at Las Campanas on 1978 Dec. 16. (8) These are the reddening corrections to be applied to the observed values. They are based on an $E(B-V)=0.17$. (9) Tifft and Snell (1971) get $E(B-V)=0.13$ for the BW field.

TABLE 3

INFRARED OBSERVATIONS OF LMC “RC” (RADIO CENTER) STARs ${ }^{a}$

\begin{tabular}{|c|c|c|c|c|c|c|c|}
\hline \multirow[b]{2}{*}{ STAR } & \multirow[b]{2}{*}{$\mathrm{Sp}$} & \multicolumn{5}{|c|}{ OBSERVED VALUES ${ }^{\mathrm{b}}$} & \multirow[b]{2}{*}{ NOTES $^{c}$} \\
\hline & & $K$ & $(J-K)$ & $(H-K)$ & $\mathrm{H}_{2} \mathrm{O}$ & $\mathrm{CO}$ & \\
\hline 2. & $\mathrm{C}$ & 10.22 & 1.79 & 0.69 & 0.175 & -0.04 & 1 \\
\hline $12 \ldots$ & $\mathrm{C}$ & 9.59 & 1.78 & 0.70 & $0.17(3)$ & -0.035 & 1 \\
\hline .......... & M6.0 & 10.41 & 1.19 & 0.30 & $0.045(3)$ & $+0.23(3)$ & 2 \\
\hline $38 \ldots$ & $\mathrm{C}$ & 10.06 & 1.73 & 0.65 & $0.14(3)$ & 0.00 & 1 \\
\hline ........ & $\mathrm{C}$ & 9.76 & 1.47 & 0.50 & $0.10(4)$ & +0.10 & 3 \\
\hline 48 . & $\mathrm{C}$ & 10.44 & 1.66 & 0.61 & $0.15(3)$ & +0.04 & 4 \\
\hline$\ldots$ & M6.5 & 10.21 & 1.28 & 0.34 & $0.12(4)$ & +0.165 & 3 \\
\hline .......... & $\mathrm{C}$ & 10.10 & 2.04 & 0.81 & & & 3 \\
\hline $54 \ldots \ldots \ldots \ldots \ldots$ & $\mathrm{C}$ & 10.34 & 1.94 & 0.81 & $0.18(4)$ & -0.055 & 3 \\
\hline Corrections ... & & -0.03 & -0.05 & -0.02 & -0.005 & 0.00 & 5 \\
\hline
\end{tabular}

${ }^{\text {a }}$ Star identification numbers and spectral types are from Blanco et al. 1980.

${ }^{\mathrm{b}}$ Photometric and statistical uncertainties do not exceed $\pm 0.02 \mathrm{mag}$ unless otherwise noted.

${ }^{\mathrm{c}}$ Notes. - (1) Observed on 1979 Nov. 2 with CTIO $4 \mathrm{~m}$ telescope. (2) Observed on 1979 Dec. 15 with Las Campanas $2.5 \mathrm{~m}$. (3) Observed on 1979 Nov. 8 with CTIO $4 \mathrm{~m}$. (4) Observed on 1979 Nov. 2 and 8 with CTIO $4 \mathrm{~m}$. (5) These are reddening corrections to be applied to the observed values. They are based on an $E(B-V)=0.09$ as discussed in the text. 
TABLE 4

INFRARED OBSERVATIONS OF SMC “B” (BAR) STARS ${ }^{\mathrm{a}}$

\begin{tabular}{|c|c|c|c|c|c|c|c|c|}
\hline \multirow{2}{*}{\multicolumn{2}{|c|}{ STAR }} & \multirow[b]{2}{*}{$\mathrm{Sp}$} & \multicolumn{5}{|c|}{ OBSERVED VALUES ${ }^{\mathrm{b}}$} & \multirow[b]{2}{*}{ NOTES $^{\mathrm{c}}$} \\
\hline & & & $K$ & $(J-K)$ & $(H-K)$ & $\mathrm{H}_{2} \mathrm{O}$ & $\mathrm{CO}$ & \\
\hline 2 & $\ldots$ & $\mathrm{C}$ & 11.37 & 1.58 & 0.58 & $0.20(4)$ & +0.025 & 1 \\
\hline 5 & $\ldots$ & $\mathrm{C}$ & 12.63 & $1.14(3)$ & 0.24 & $\ldots$ & & 2 \\
\hline 8 & $\ldots$ & $\mathrm{C}$ & 11.37 & 1.21 & 0.34 & $0.10(4)$ & +0.12 & 1 \\
\hline 10 . & $\ldots$ & $\mathrm{C}$ & 11.75 & 1.07 & 0.26 & $0.06(3)$ & $+0.17(3)$ & 2 \\
\hline 13. & $\ldots$ & C & 11.14 & 1.55 & 0.57 & $0.17(3)$ & -0.01 & 2 \\
\hline 18. & $\ldots$ & $\mathrm{C}$ & 11.84 & $1.78(3)$ & 0.68 & $0.25(3)$ & -0.05 & 2 \\
\hline 22. & $\ldots$ & $\mathrm{C}$ & 11.24 & 1.33 & 0.50 & & & 3 \\
\hline 23 . & $\ldots$ & C & 10.24 & $1.69(3)$ & 0.62 & 0.22 & -0.005 & 4 \\
\hline 24 & $\ldots$ & $\mathrm{C}$ & $12.03(3)$ & $1.13(4)$ & $0.27(3)$ & & & 5 \\
\hline 25. & $\ldots$ & $\mathrm{C}$ & 11.33 & 1.74 & 0.70 & $0.19(4)$ & -0.06 & 1 \\
\hline 28 & $\ldots$ & M6.0 & 9.38 & 1.26 & 0.44 & 0.62 & +0.14 & 3,6 \\
\hline 30 & $\ldots$ & $\mathrm{C}$ & 10.48 & 1.18 & 0.36 & $0.09(4)$ & +0.13 & 1 \\
\hline 31 & $\ldots$ & $\mathrm{C}$ & 10.32 & 1.55 & 0.56 & $0.15(4)$ & +0.01 & 1 \\
\hline 36 & $\ldots$ & $\mathrm{C}$ & 11.50 & 1.43 & 0.52 & $0.165(3)$ & +0.05 & 2 \\
\hline 39 & $\ldots$ & $\mathrm{C}$ & 10.60 & 1.77 & 0.69 & $0.17(3)$ & -0.06 & 2 \\
\hline 40 & $\ldots$ & $\mathrm{C}$ & 11.20 & $1.73(4)$ & 0.67 & $0.21(4)$ & $-0.05(3)$ & 5 \\
\hline 45 & $\ldots$ & $\mathrm{C}$ & $11.19(3)$ & $1.40(4)$ & $0.49(3)$ & & & 5 \\
\hline 47 & $\ldots$ & $\mathrm{C}$ & $10.74(3)$ & $1.50(3)$ & $0.54(3)$ & $0.12(4)$ & $+0.005(3)$ & 3,4 \\
\hline 52 & $\ldots$ & C & 11.12 & 1.63 & 0.65 & 0.17 (3) & -0.065 & 2 \\
\hline 65 & $\ldots$ & $\mathrm{C}$ & 10.21 & 1.63 & 0.64 & $0.15(4)$ & -0.04 & 1 \\
\hline \multirow[t]{2}{*}{74} & $\ldots$ & $\mathrm{C}$ & $10.42(3)$ & $1.65(3)$ & 0.56 & $0.165(3)$ & $+0.05(3)$ & 4 \\
\hline & \multicolumn{2}{|c|}{ Corrections } & -0.03 & -0.06 & -0.02 & -0.005 & +0.005 & 7 \\
\hline
\end{tabular}

${ }^{a}$ Star identification numbers and spectral types are from Blanco et al. 1980.

${ }^{b}$ Numbers in parentheses are combined photometric and statistical uncertainties in units of hundreths of a magnitude when greater than 2.

'NOTES. - (1) Observed on 1979 Nov. 8 with CTIO 4 m telescope. (2) Observed on 1979 Nov. 9 with CTIO $4 \mathrm{~m}$. (3) Observed on 1978 Dec. 20 with Las Campanas $2.5 \mathrm{~m}$. (4) Observed on $1979 \mathrm{Dec}$. 31 with Las Campanas $2.5 \mathrm{~m}$. (5) Observed on $1980 \mathrm{Jan}$. 1 with Las Campanas $2.5 \mathrm{~m}$. (6) Other observations: 1979 Nov. $9, K=9.93, J-K=1.32, H-K=0.55, \mathrm{H}_{2} \mathrm{O}=0.76, \mathrm{CO}=0.10 .1979$ Dec. $31, K=9.83, J-K=1.12, H-K=0.47$, $\mathrm{H}_{2} \mathrm{O}=0.52, \mathrm{CO}=0.00$. See text for discussion. (7) These are reddening corrections to be applied to the observed values. They are based on an $E(B-V)=0.11$ as discussed in the text.

variability class. This association of red color with large amplitude variability is again consistent with the results of Frogel and Hyland.

Figure 2 compares the $J H K$ colors of the LMC and SMC samples of $C$ and $M$ stars with those of their galactic counterparts. The trends in colors are similar for the various samples of stars, but small and apparently real differences do exist. In Figure 2 we see a tendency at fixed $(H-K)_{0}$ for the SMC C stars to be $0.05 \mathrm{mag}$ bluer in $(J-H)_{0}$ than the LMC stars, while the galactic $\mathrm{C}$ stars are $0.06 \mathrm{mag}$ redder in $(J-H)_{0}$. The strong separation between LMC and SMC carbon stars in $(\mathrm{J}-\mathrm{H})_{0}$ and $(\mathrm{H}-\mathrm{K})_{0}$ suggested in Figure 2 of Mould and Aaronson (1980) is an exaggeration based on too small a sample.

\section{ii) Narrow Band Indices}

The dependence of the CO index on $(J-K)_{0}$ for the galactic $C$ stars is displayed in Figure 3. A least squares line was fitted to the data in the region $1.2 \leq(J-K)_{0} \leq$ 2.0 in the same manner as described above. The data in Figure 3 display a scatter which is large and presumably intrinsic. A similar scatter and dependence on $J-K$ is seen in the high-resolution $\mathrm{CO}$ measurements of Frogel and Hyland (1972).

Figure 4 shows the $\left[\mathrm{CO},(J-K)_{0}\right]$-diagram for the Magellanic Cloud $C$ and $M$ stars, together with the least squares fit for the galactic $\mathrm{C}$ star sample transferred from Figure 3 . The trend in $\mathrm{CO}$ index with $J-K$ color for the LMC and SMC stars is similar to that in Figure 3 , but at a given $(J-K)_{0}$ the galactic $\mathrm{C}$ stars have $\mathrm{CO}$ indices that are systematically $0.06 \mathrm{mag}$ greater than those of the LMC C stars, while the SMC C stars are perhaps 0.02 mag weaker than those in the LMC. There also appears to be a difference in $\mathrm{CO}$ indices between the $\mathrm{BMB}$ and WORC samples. However, because of strong selection effects in the latter sample, we shall not consider this difference further.

Figure 5 shows there is a tight relationship between the $\mathrm{H}_{2} \mathrm{O}$ index and $(J-K)_{0}$ for galactic $\mathrm{C}$ stars. As noted in Frogel, Persson, and Cohen (1980) and discussed in $\S \mathrm{V} b$ here, $\mathrm{C}$ stars do not have any water absorption, and the $\mathrm{H}_{2} \mathrm{O}$ index is primarily a measure of continuum slope. A least squares line was fitted to the 
TABLE 5

INFRARED OBSERVATIONS OF WESTERLUND et al. LMC CARBON STARS ${ }^{\mathrm{a}}$

\begin{tabular}{|c|c|c|c|c|c|c|c|}
\hline \multirow[b]{2}{*}{ STAR } & \multirow[b]{2}{*}{ Sp } & \multicolumn{5}{|c|}{ OBSERVED Values ${ }^{b}$} & \multirow[b]{2}{*}{ NOTES $^{c}$} \\
\hline & & $K$ & $(J-K)$ & $(H-K)$ & $\mathrm{H}_{2} \mathrm{O}$ & $\mathrm{CO}$ & \\
\hline $40 \ldots$. & $\mathrm{C} 4,4$ & 9.89 & 1.45 & 0.46 & 0.12 & +0.135 & 1 \\
\hline $46 \ldots$ & $\mathrm{C}, 3 \mathrm{e}$ & 9.63 & 1.77 & 0.66 & 0.195 & -0.03 & 1,7 \\
\hline $47 \ldots$. & $\mathrm{C} 3,5$ & $10.31(3)$ & 1.31 & 0.38 & 0.14 & +0.20 & 1 \\
\hline $56 \ldots$ & $\mathrm{C} 5,3$ & $10.12(3)$ & 1.31 & 0.35 & 0.07 & +0.20 & 1 \\
\hline $64 \ldots$ & C5,5 & 10.86 & $1.36(4)$ & $0.42(3)$ & $0.12(3)$ & $+0.09(3)$ & 2 \\
\hline $65 \ldots$ & $\mathrm{C} 7,2 \mathrm{~J}$ & 10.17 & $1.27(4)$ & $0.35(3)$ & 0.155 & +0.285 & 2 \\
\hline $67 \ldots$ & $\mathrm{C} 4,2 \mathrm{~J}$ & 10.10 & $0.94(4)$ & 0.22 & 0.10 & +0.13 & 2 \\
\hline $78 \ldots$ & $\mathrm{C} 4,4 \mathrm{~J}$ & $10.12(3)$ & 1.05 & 0.27 & 0.08 & +0.23 & 1 \\
\hline $84 \ldots$ & C4,3J & $9.50(3)$ & 1.08 & 0.26 & 0.095 & +0.175 & 1 \\
\hline $105 \ldots$ & $\mathrm{C} 4,5$ & 10.30 & $1.72(3)$ & 0.62 & 0.185 & -0.035 & 2,7 \\
\hline $106 \ldots$ & $\mathrm{C} 4,4 \mathrm{~J}$ & 9.63 & $1.27(4)$ & $0.38(3)$ & 0.115 & +0.11 & 2 \\
\hline $107 \ldots$ & $\mathrm{C}, 4$ & 10.22 & $1.55(3)$ & 0.56 & 0.095 & -0.05 & 3 \\
\hline $108 \ldots$ & $\mathrm{C}, 3$ & 9.85 & $1.59(3)$ & 0.57 & 0.16 & -0.005 & 3 \\
\hline $112 \ldots$ & $\mathrm{C} 4,3$ & 10.01 & $1.13(3)$ & 0.28 & 0.075 & +0.09 & 4 \\
\hline $131 \ldots$ & $\mathrm{C} 4,5 \mathrm{~J}$ & 9.96 & $1.50(3)$ & 0.51 & 0.17 & -0.01 & 4 \\
\hline $137 \ldots$ & $\mathrm{C}, 5$ & 10.19 & $1.41(3)$ & 0.42 & 0.12 & +0.14 & 4 \\
\hline $157 \ldots$ & $\mathrm{C} 5,3$ & 10.66 & $1.49(3)$ & 0.47 & 0.09 & +0.17 & 4 \\
\hline $186 \ldots$ & $\mathrm{C} 6,2 \mathrm{e}$ & 9.12 & $2.21(3)$ & 1.10 & 0.20 & -0.10 & 4 \\
\hline $190 \ldots$ & $\mathrm{C} 4,4$ & 10.14 & $1.40(3)$ & 0.42 & 0.095 & +0.21 & 4 \\
\hline $196 \ldots$ & $\mathrm{C} 4,4$ & 10.37 & $1.35(4)$ & 0.42 & 0.075 & +0.085 & 5 \\
\hline $214 \ldots$ & $\mathrm{C} 4,3$ & 10.11 & $1.23(3)$ & 0.34 & 0.085 & +0.18 & 5 \\
\hline $231 \ldots$ & & 10.15 & $1.77(3)$ & 0.67 & $0.21(3)$ & $-0.03(3)$ & 6 \\
\hline $239 \ldots$ & $\mathrm{C}, 3$ & 10.33 & $1.50(3)$ & 0.51 & $0.15(3)$ & $+0.06(3)$ & 6,8 \\
\hline $256 \ldots$ & $C 7,3 \mathrm{e}$ & 11.15 & $1.30(3)$ & 0.43 & $0.06(3)$ & $+0.075(4)$ & 6 \\
\hline $272 \ldots$ & $\mathrm{C}, 5$ & 10.30 & $1.46(3)$ & 0.51 & $0.035(3)$ & $+0.03(3)$ & 6 \\
\hline $300 \ldots$ & $\mathrm{C}, 5$ & 10.27 & $1.66(3)$ & 0.64 & $0.225(3)$ & $+0.025(3)$ & 6 \\
\hline \multicolumn{2}{|c|}{ Corrections } & -0.03 & -0.07 & -0.02 & -0.005 & +0.005 & 9 \\
\hline
\end{tabular}
${ }^{\mathrm{a}} \mathrm{T}$.

${ }^{\mathrm{b}}$ Statistical uncertainties in colors, magnitudes, and indices are $\pm 0.02 \mathrm{mag}$ or less, unless otherwise noted. ${ }^{c}$ Notes.-(1) Observed on 1978 Dec. 8 with Las Campanas $2.5 \mathrm{~m}$ telescope. (2) Observed on 1978 Dec. 9 with Las Campanas $2.5 \mathrm{~m}$. (3) Observed on $1978 \mathrm{Dec}$. 10 with Las Campanas $2.5 \mathrm{~m}$. (4) Observed on 1978 Dec. 11 with Las Campanas $2.5 \mathrm{~m}$. (5) Observed on 1978 Dec. 13 with Las Campanas $2.5 \mathrm{~m}$. (6) Observed on 1978 Dec. 14 with Las Campanas $2.5 \mathrm{~m}$. (7) Also observed with CTIO $4 \mathrm{~m}$ on $1978 \mathrm{Dec}$. 19. (8) Star W46 in NGC 2209. (9) These are the reddening corrections to be applied to the observed values. They are based on a mean $E(B-V)=0.12$ as discussed in text.

data in the region $1.2 \leq(J-K)_{0} \leq 2.0$ in the same manner as described above. As for the $\left[(J-H)_{0},(H-K)_{0}\right]-$ relationship, we again see the observed scatter could be due entirely to a combination of uncertainties in the reddening correction and in the measurements themselves.

The correlation of $\mathrm{H}_{2} \mathrm{O}$ index with $(J-K)_{0}$ for the LMC and SMC stars is shown in Figure 6, where the least squares fit to the galactic $C$ star sample has been transferred from Figure 5. To within the uncertainties of the measurements, there is no difference in the $\mathrm{H}_{2} \mathrm{O}-(J$ $-K)_{0}$ relation for the galactic, LMC and SMC samples.

\section{iii) Infrared Color-Magnitude Diagram}

Figure 7 is the color-magnitude diagram for all $\mathrm{C}$ and $M$ stars observed in the Magellanic Clouds. The SMC stars have been shifted brighter by $0.5 \mathrm{mag}$, which corresponds to the mean difference in $m_{I}$ found by BMB. Essentially all of the $C$ stars plotted are brighter than the tips of the giant branches of Galactic globular clusters.

The SMC stars occupy the same magnitude regime as the LMC carbon stars from the BMB survey, although the former do not appear to extend to as red a color, qualitatively consistent with BMB's finding that the mean $R-I$ color of the SMC stars is 0.2-0.3 mag bluer than that of the LMC stars. The WORC carbon stars extend to considerably bluer colors and have a tendency to be somewhat brighter than the BMB stars. This result is not surprising in view of the vastly different nature of the two surveys as discussed by BMB. In particular, Figure 7 is entirely consistent with BMB's contention that the WORC carbon stars merely represent the bright tail of the distribution of carbon stars which BMB found, and not a separate population. Figure 7 does not 


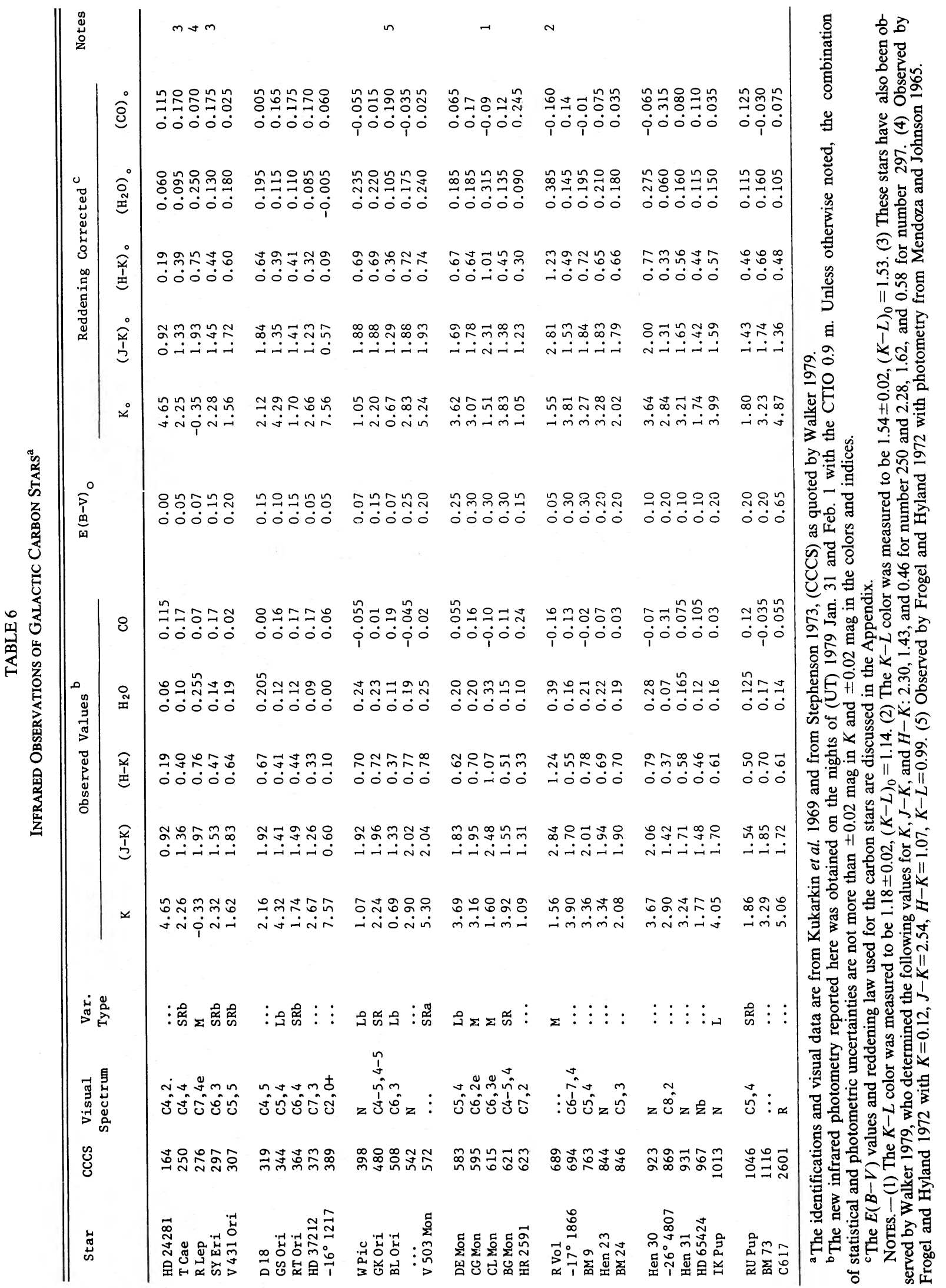




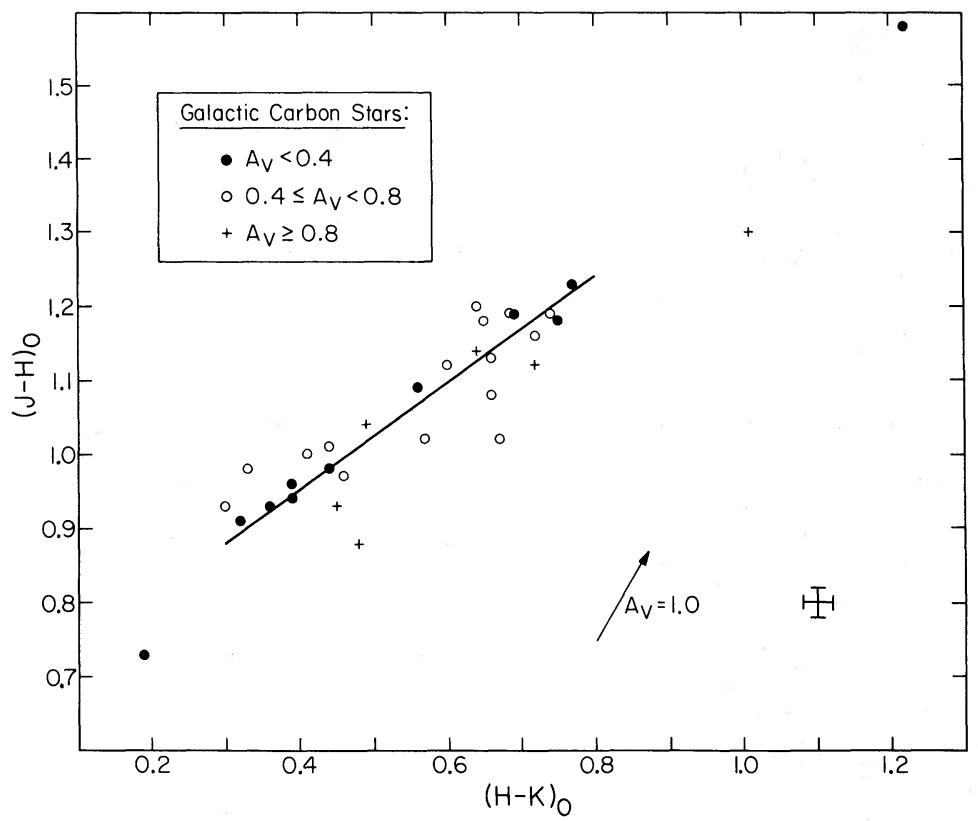

Fig. 1.- $(J-H)_{0}$ is shown as a function of $(H-K)_{0}$ for the sample of galactic carbon stars. The filled circles, open circles, and crosses represent stars having $A_{V}<0.4,0.4 \leq A_{V} \leq 0.8$, and $A_{V}>0.8 \mathrm{mag}$, respectively. An error bar for each point is shown in the lower right, as is a reddening vector for $A_{V}=1.0 \mathrm{mag}$. The least squares fit to the data, with double weight given to the stars with $A_{V}<0.4 \mathrm{mag}$, is $(J-H)_{0}=0.72(H-K)_{0}+0.66$, and is shown as the solid line.

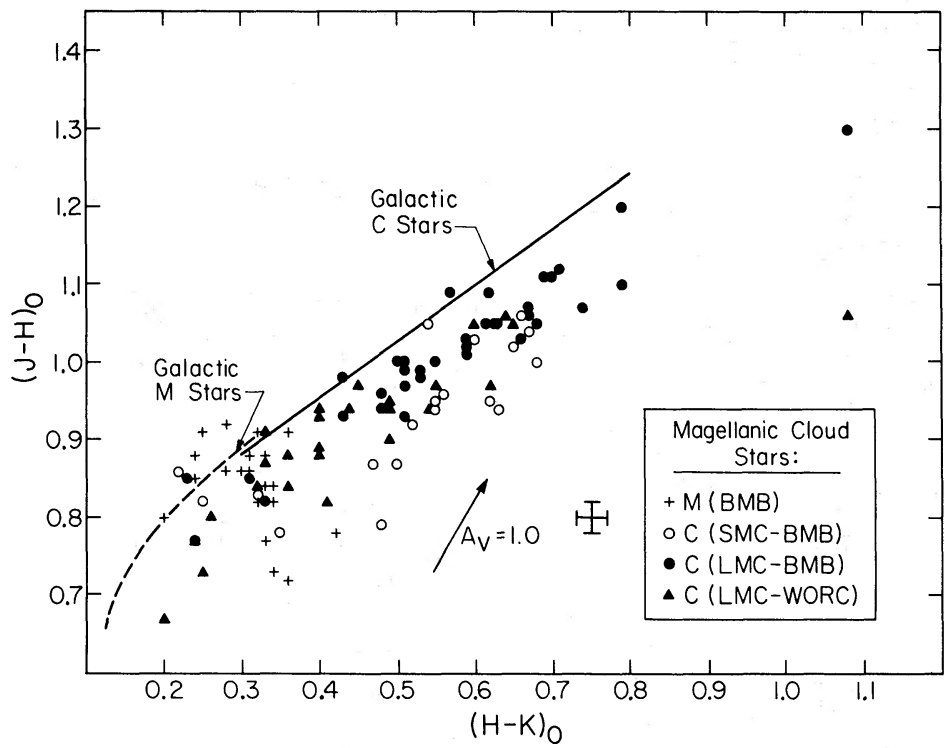

FIG. 2.- $(J-H)_{0}$ is shown as a function of $(H-K)_{0}$ for the SMC carbon stars from the BMB survey (open circles), the LMC carbon stars from the BMB survey (filled circles), the LMC carbon stars from the WORC survey (filled triangles), and the M stars of the BMB survey (crosses). An observational error typical of each point is shown in the lower right. The mean line for galactic $M$ stars is plotted from Frogel et al. (1978), and the mean relationship for galactic carbon stars is that of Fig. 1. 


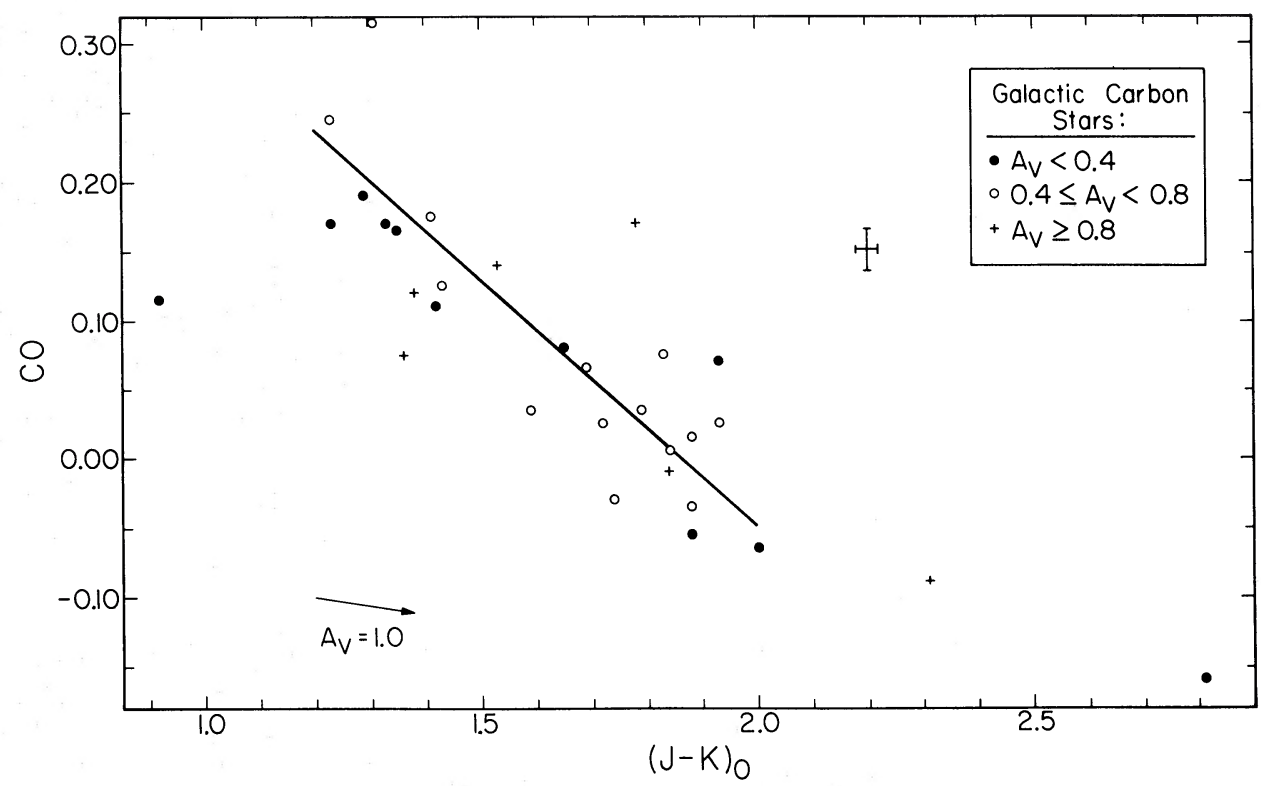

FIG. 3.- The $\mathrm{CO}$ index is shown as a function of $(J-K)_{0}$ for the sample of galactic carbon stars. The symbols have the same meaning as in Fig. 1. An error bar for each point is at the upper right, while a reddening vector for $A_{V}=1.0 \mathrm{mag}$ is at the lower left. The least squares fit to the galactic data with double weight for stars with $A_{V}<0.4 \mathrm{mag}$ is $\mathrm{CO}=-0.36(J-K)_{0}+0.67$ and is shown by the solid line.

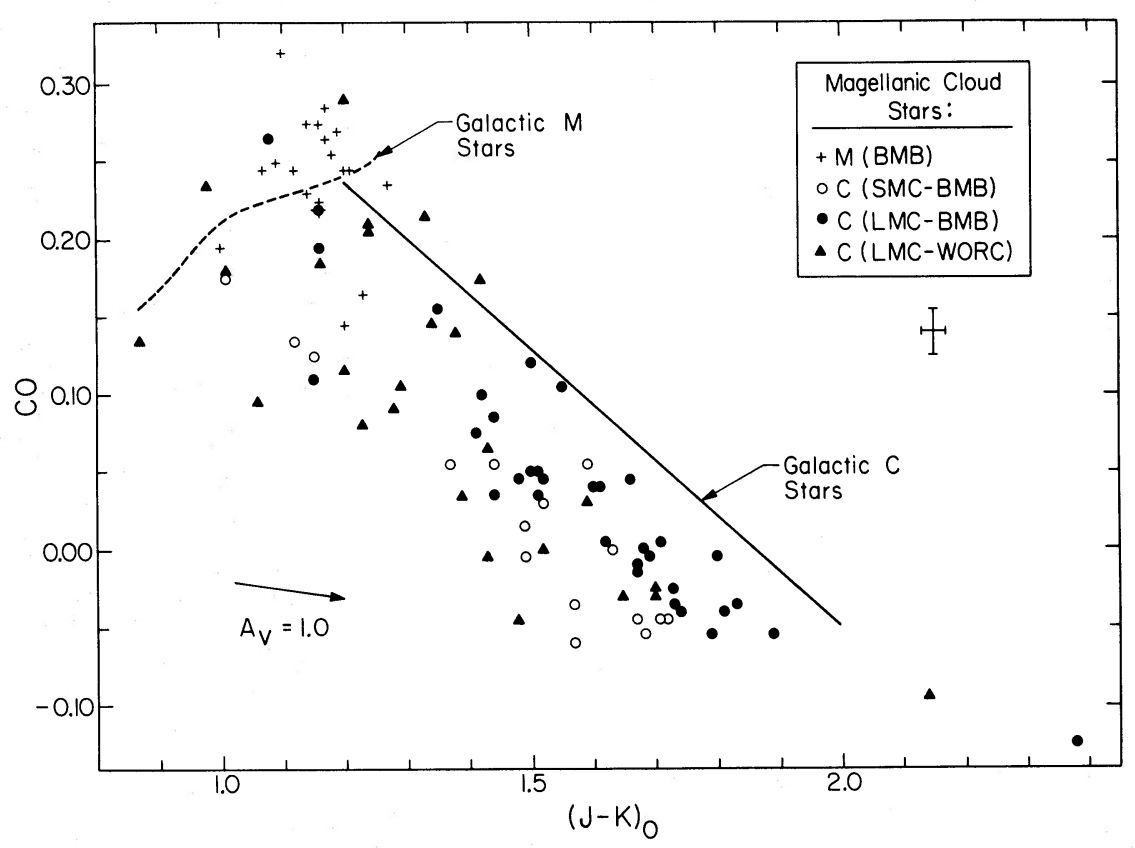

FIG. 4. - The CO index is shown as a function of $(J-K)_{0}$ for the Magellanic Cloud carbon stars of the BMB and WORC surveys and for the M stars of the BMB survey. The symbols are as in Fig. 2. An error bar typical of each point is shown at the upper right. The mean line for galactic M stars (short-dashed line) is from Frogel et al. (1978), while the mean relationship for galactic carbon stars is that of Fig. 3. 


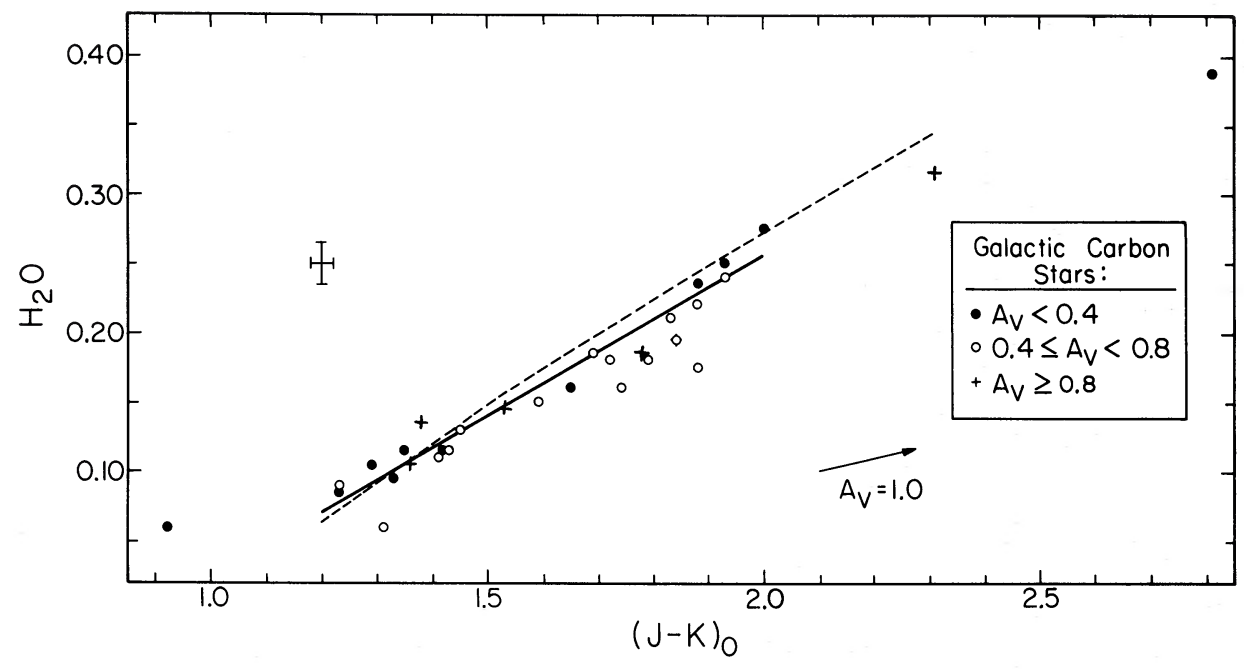

FIG. 5. - The $\mathrm{H}_{2} \mathrm{O}$ index is plotted as a function of $(J-K)_{0}$ for the sample of galactic carbon stars. The symbols are as in Fig. 1. An error bar for each point is at the upper left, and a reddening vector for $A_{V}=1.0 \mathrm{mag}$ is at the lower right. The solid line is the least squares fit to the data with double weight assigned to stars with $A_{V}<0.4$ mag: $\mathrm{H}_{2} \mathrm{O}=0.23(J-K)_{0}-0.205$. The light dashed line is the relationship between $\mathrm{H}_{2} \mathrm{O}$ and $(J-K)_{0}$ predicted from $H-K$ colors as described in $\S \mathrm{V} b$.

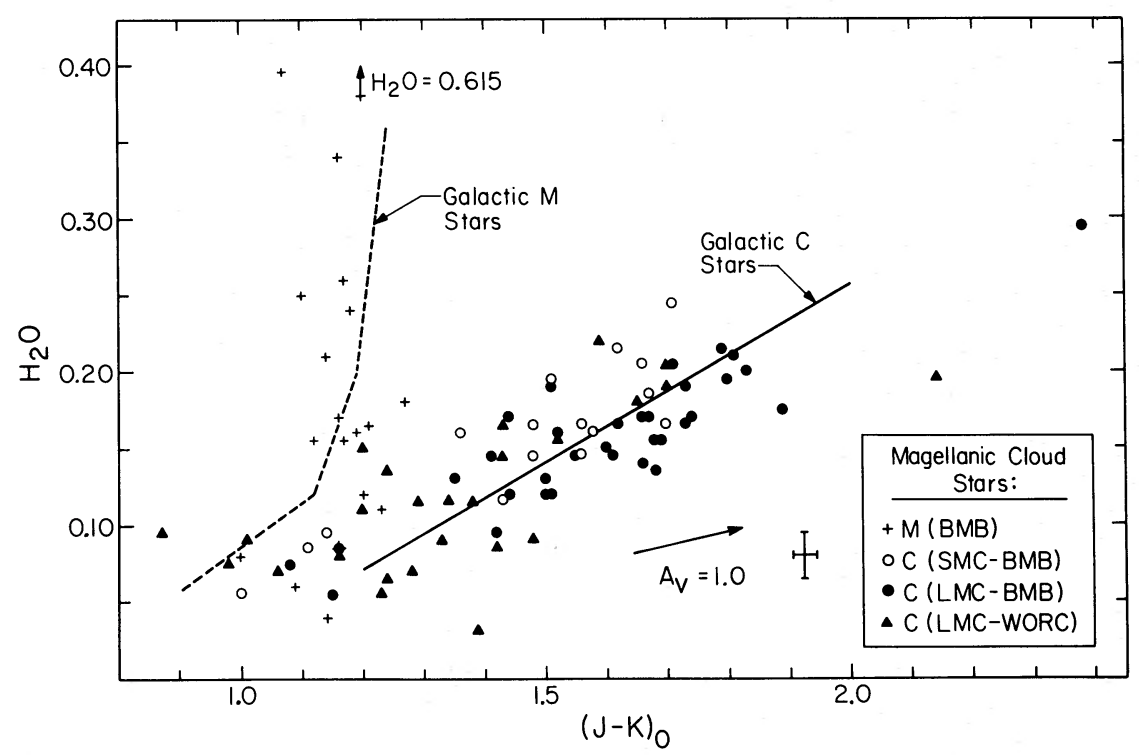

FIG. 6. - The $\mathrm{H}_{2} \mathrm{O}$ index is shown as a function of $(J-K)_{0}$ for the Magellanic Cloud carbon stars of the BMB and WORC surveys and for the M stars of the BMB survey. The symbols are as in Fig. 2. An error bar typical of each point is at the lower right. The short-dashed line is the mean relationship for galactic M giants from Aaronson et al. (1978), while the mean line for galactic carbon stars is that of Fig. 5 . 


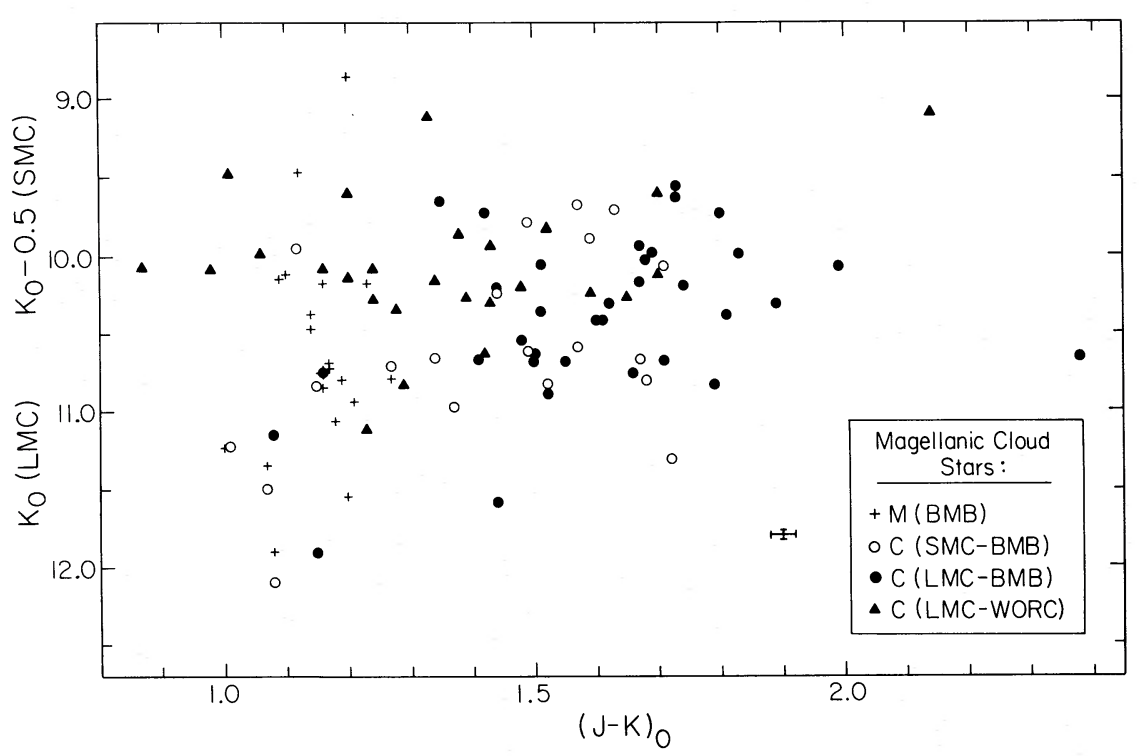

FIG. 7.- The $K_{0}$ magnitudes are shown as a function of $(J-K)_{0}$ for the magellanic Cloud carbon stars of the BMB and WORC surveys and for the M stars of the BMB survey. The symbols are as in Fig. 2. The SMC stars have been offset by $0.5 \mathrm{mag}$ in $K_{0}$ to allow for the difference in distance modulus from that of the LMC.

display any obvious color-magnitude relation for either the $\mathrm{M}$ or the $\mathrm{C}$ stars. This is particularly true for the $\mathrm{C}$ stars, which span a range of $1.5 \mathrm{mag}$ in $(J-K)_{0}$.

\section{b) The M Stars}

Our data on several $\mathrm{M}$ stars of the BMB survey have also been plotted in Figures 2, 4, and 6 together with the mean relationships for their galactic counterparts from Frogel et al. (1978) and Aaronson, Frogel, and Persson (1978). Unfortunately, for the latest spectral type galactic $M$ stars, these colors and indices are not well known (Frogel et al. 1978), and the scatter in the colors and indices in Figures 2, 4, and 6 may also be present in samples of M stars in the Galaxy.

At a given $(J-K)_{0}$ the Magellanic Cloud M stars tend to have stronger $\mathrm{H}_{2} \mathrm{O}$ indices, in the mean, than their galactic counterparts. This may be the reason why they also have redder $(H-K)_{0}$ colors at a given $(J-H)_{0}$ than the galactic stars (see below). However, the galactic M star data have not been corrected for reddening, which for such high-luminosity, rare objects may be as much as $0.05 \mathrm{mag}$ in $J-K$, enough to eliminate these small differences. Although the LMC is probably slightly metal poor compared to the Galaxy, the extreme saturation of the first overtone $\mathrm{CO}$ bands would prevent the $\mathrm{CO}$ index from changing by a detectable amount between these two groups of stars. The three Magellanic Cloud M stars with the largest $\mathrm{H}_{2} \mathrm{O}$ indices are among the five stars which lie the furthest below and to the right of the mean $\left[(J-H)_{0},(H-K)_{0}\right]-$ line. This is reminiscent of the situation for the variables in 47 Tuc (Frogel, Persson, and Cohen 1981), where we argued that $\mathrm{H}_{2} \mathrm{O}$ absorption was affecting their $J H K$ colors.
In the color-magnitude diagram of Figure 7 , the M stars found by BMB have a range in $K_{0}$ of at least 2 mag but do not extend redward of $(J-K)_{0}=1.30$. At a given magnitude, the $M$ stars exhibit a spread in $(J-K)_{0}$ which is most likely real. With the exception of SMC-B28, which is unusual in several other respects (see $\S \mathrm{IV} c$ ), the bright limit of the M stars is generally the same as or somewhat fainter than that of the C stars.

\section{c) Cluster and Field Star Comparison}

Infrared data for $C$ and $M$ stars in clusters of the LMC and SMC have been presented by Mould and Aaronson (1980) and Frogel, Persson, and Cohen (1980, hereafter FPC). The number of data on cluster stars is not yet very extensive, but what is available does indicate a general similarity in the infrared properties of these stars and those of the Magellanic Cloud field. In particular, the area of the color-magnitude diagram (Fig. 7) populated by field Magellanic Cloud C stars includes within it the observed LMC and SMC cluster C stars. There is no obvious difference in mean $K$ magnitude between the field and cluster stars. This result cannot be extended to the M stars because the BMB survey does not include stars of spectral type M5 and earlier.

\section{THE LUMINOSITY FUNCTION OF LUMINOUS STARS IN THE MAGELLANIC CLOUDS}

\section{a) Derivation of Bolometric Luminosities}

As mentioned in the Introduction, the BMB C star sample is complete except for those with weak $\mathrm{CN}$ absorption (see BMB for details); the faintest C star 
found is 1.5 mag brighter than the survey limit. We can thus obtain for the first time an unbiased luminosity function for carbon stars which can be compared to theoretical predictions. The onset of the carbon star phase in an asymptotic giant branch (AGB) star is a sensitive function of the physics of the dredge-up process, nuclear reactions, metal abundance, etc. Given the severe uncertainties in the modeling of these processes, a comparison of the theoretical and observed luninosity functions may lead to important constraints on theoretical parameters.

The stars with infrared photometry constitute $17 \%$ of those discovered by BMB, but are in fact biased toward the bright end of the distribution. Because of the extremely red color of $\mathrm{C}$ stars, it is much easier and more accurate to derive bolometric corrections using infrared rather than visual data. BMB provide $I$ magnitudes and $R-I$ colors; from our subset with $J H K$ photometry we derive correlations to convert $I$ magnitudes into bolometric magnitudes for the entire BMB sample. Bolometric corrections to the $K$ magnitude $\mathrm{BC}_{K}$, as a function of $J-K$ color, based on broad band photometry from $U$ to $L$ of galactic C stars by Mendoza and Johnson (1965), are given in FPC for carbon stars. In § III we showed that although there are differences in the $J H K$ colors between the Magellanic Cloud and galactic $\mathrm{C}$ stars, these differences are small. For $(J-K)_{0}>1.0 \mathrm{mag}$, as is the case for almost all of the SMC and LMC carbon stars, $\mathrm{BC}_{K}$ is not very sensitive to $(J-K)_{0}$; a $0.1 \mathrm{mag}$ error in $(J-K)_{0}$ produces a maximum error of $0.1 \mathrm{mag}$ in $\mathrm{BC}_{K} \cdot{ }^{3}$ We have thus computed $m_{\mathrm{bol}}$ for the $\mathrm{BMB}$ carbon stars from the data in Tables 1-4. In Figure 8 we plot $m_{I_{0}}-m_{\text {bol }}$ for these carbon stars, where $m_{I}$ is taken from $\mathrm{BMB}$, as a function of $(R-I)_{0}$, and $R-I$ is on the Kron-Cousins system. For four stars in the LMC $\mathrm{BW}$ field, $\mathrm{BMB}$ do not give $R-I$ colors, and their colors were derived by transforming Richer's (1981) $R$ and $I$ photoelectric photometry in the Johnson System.

The relationship of Figure 8 for the SMC carbon stars is well defined, with a scatter consistent with the measurement uncertainties except for four stars marked with crosses. An inspection of BMB's original plates indicated that one of these (SMC-B45) is a large amplitude variable; two (SMC-B18 and B40) vary over at least 0.3 mag at $I$; the remaining discrepant star (SMC-B22) is a

\footnotetext{
${ }^{3}$ The effect on $\mathrm{BC}_{K}$ of the shift in the $J H K$ colors of the Magellanic Cloud stars compared with those in the Galaxy, upon which the bolometric corrections of FPC are based, was checked by computing $\mathrm{BC}_{K}$ directly for all the stars with VRIJHK photometry from the WORC sample and Richer (1981). The systematic difference in $\mathrm{BC}_{K}$ between the Magellanic Cloud and the galactic carbon stars read at a given $(J-K)_{0}$ amounts to at most 0.05 mag at all $(J-K)_{0}$. Thus all the bolometric luminosities in this paper could be adjusted fainter by small amounts; we have, however, not done so, on the grounds that there are surely other systematic effects present which we cannot investigate from broad band photometry alone.
}

close double and the $I$ magnitude is suspect. The least squares fits with $m_{I_{0}}-m_{\text {bol }}$ and with $(R-I)_{0}$ as independent variables, omitting the four discrepant SMC stars, were averaged to give:

$$
m_{I_{0}}-m_{\mathrm{bol}}=-1.27+1.42(R-I)_{0}, \quad \text { for the SMC, }
$$

with a correlation coefficient of 0.87 . The sense of the correlation between $m_{I_{0}}-m_{\text {bol }}$ and $(R-I)_{0}$ is that expected for redder $(R-I)_{0}$ colors accompanied by stronger molecular line blanketing at $I .(R-I)_{0}$ for both the SMC and LMC carbon stars correlates well with $(J-K)_{0}$ with an offset of $0.3 \mathrm{mag}$ between the two galaxies, so that for a given $(J-K)_{0}$ the LMC carbon stars are $0.3 \mathrm{mag}$ redder in $(R-I)_{0}$ than those of the SMC. This difference is presumably related to the higher mean metallicity of the LMC than the SMC.

The LMC carbon stars show a larger scatter in Figure 8. The four highly discrepant LMC stars toward the lower right of Figure 8 marked with crosses were checked for variability on BMB's original plates. Two were highly variable (LMC-0-86 and $\mathrm{BW}-1$ ), while the remaining two did not appear to vary between the two available plates. However, one of these two has a highly uncertain $R-I$ color, which was in fact not measured by $\mathrm{BMB}$, but was transformed from Richer's (1981) photometry where it was listed as a low accuracy measurement. We have therefore adopted the slope of the SMC relationship (eq. [1a]) and shifted it so as to fit through the centroid of the LMC data, to give:

$$
m_{I_{0}}-m_{\text {bol }}=-1.85+1.42(R-I)_{0}, \quad \text { for the LMC. }
$$

If the offset of $(R-I)_{0}$ is, as discussed above, due to metallicity effects, the scatter in the LMC data would indicate a range of metallicity within the LMC itself. It could also result from the inclusion of many smallamplitude variables as no systematic search for variables was made. The probable dependence of $m_{I_{0}}-M_{\text {bol }}$ on $(R-I)_{0}$ implies that the common practice of adding a constant to $m_{I}$ to obtain $m_{\text {bol }}$ (used, for example, by Richer, Olander and Westerlund 1979) can introudce systematic errors when applied to individual stars.

From equations (1a) and (lb) we obtain bolometric magnitudes as a function of $m_{I_{0}}$ and $(R-I)_{0}$ for the full BMB sample (with the exception of a small number of stars with no $R-I$ colors). The use of equations (1a) and (lb), as compared to setting $m_{I_{0}}-m_{\text {bol }}$ a constant, does not produce noticeable differences in any property of the resulting carbon star luminosity function. The bolometric luminosity distribution for the LMC and SMC C stars of the complete BMB sample is shown in Figure 9, where the SMC distribution has been shifted by 0.5 


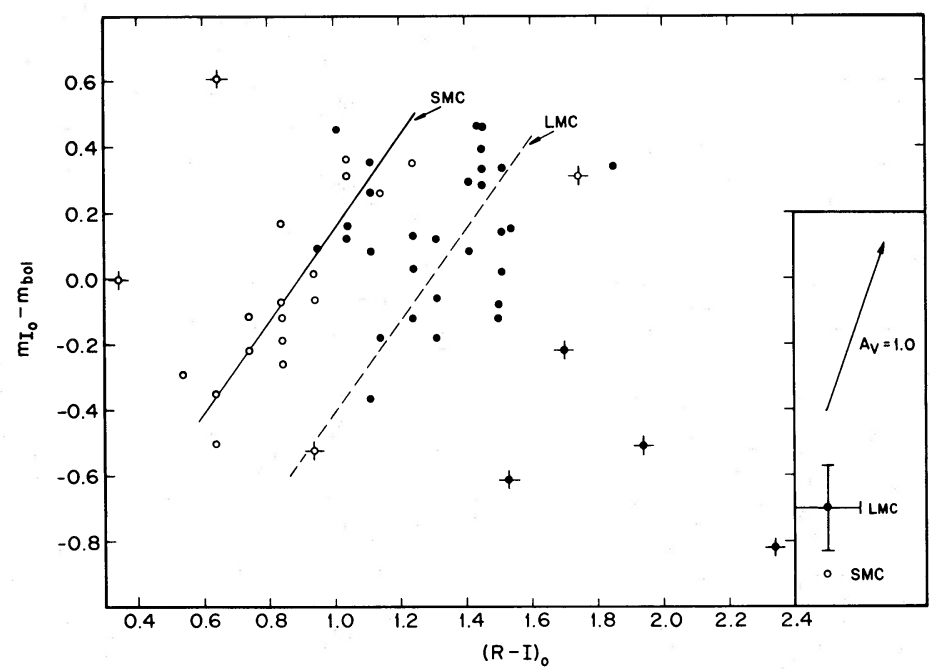

FIG. 8. - The quantity $m_{I}-m_{\text {bol }}$ is shown as a function of $(R-I)_{0}$ for the LMC carbon stars ( filled circles) and the SMC carbon stars (open circles) of the BMB sample with infrared data. The error bar in the lower right corner is typical of all the points plotted. A reddening vector for $1 \mathrm{mag}$ of visual absorption is shown. The least squares fits to the LMC and the SMC data are indicated by the solid and dashed lines, respectively.

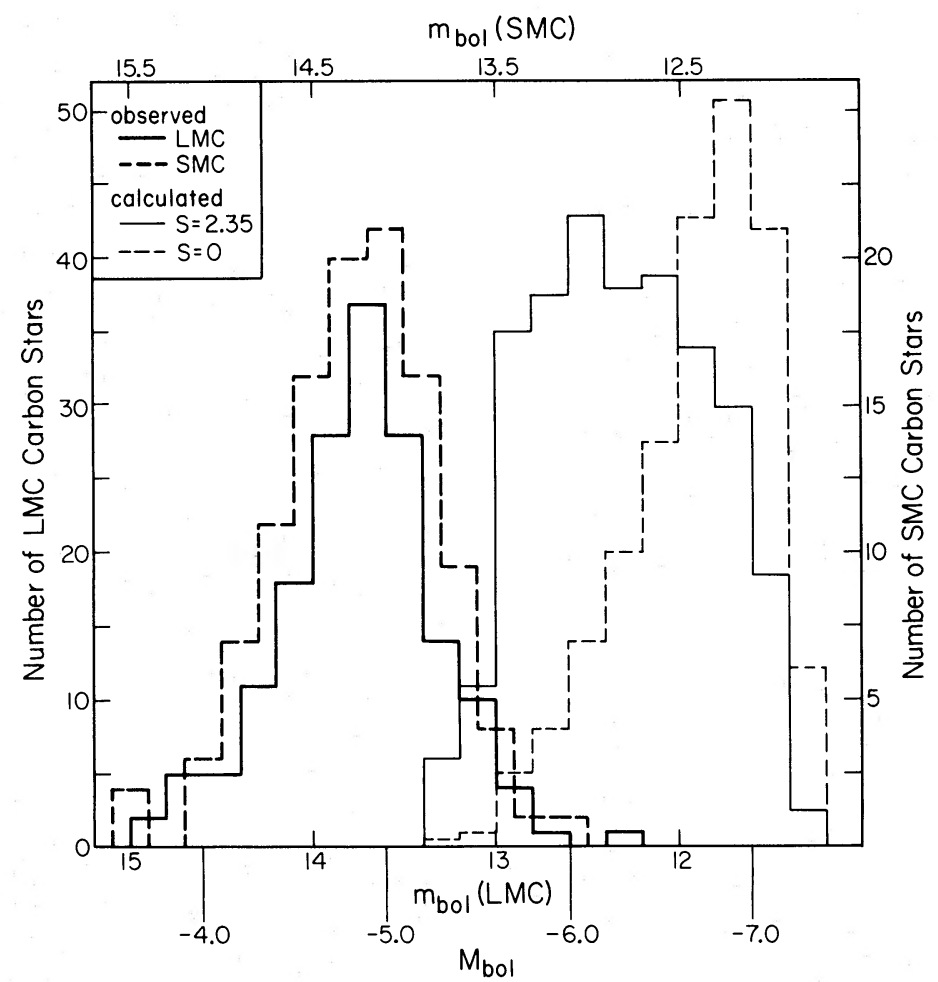

FIG. 9. - The luminosity function of the LMC (heavy solid line) and SMC (heavy dashed line) carbon stars in BMB's complete sample are shown. The predicted luminosity function of low-metallicity carbon stars from Renzini and Voli's (1980) theoretical evolutionary tracks is indicated for an initial mass function $s=2.35$ (light solid line) and $s=0.0$ (light dashed line); see text for discussion of these luminosity functions. 
TABLE 7

MEAN BOLOMETRIC LUMINOSITIES OF Magellanic Cloud Carbon Stars

\begin{tabular}{ccccc}
\hline \hline Sample & No. of Stars & Mag & Mean & $\sigma$ \\
\hline \multicolumn{7}{c}{ LMC } \\
\hline BMB ......... & 186 & $m_{I}$ & 13.98 & 0.41 \\
BMB + eq. (1) .. & 164 & $m_{\text {bol }}$ & 13.75 & 0.43 \\
\hline \multicolumn{7}{c}{ SMC } \\
\hline BMB ......... & 134 & $m_{I}$ & 14.49 & 0.45 \\
BMB + eq. (1) .. & 111 & $m_{\text {bol }}$ & 14.26 & 0.49 \\
\hline
\end{tabular}

mag, corresponding to the difference in distance modulus (BMB).

Table 7 gives the mean values of $m_{\mathrm{bol}}$ and $m_{I}$ for the BMB sample. We note that the $m_{\text {bol }}$ distribution of Figure 9 is very similar to the BMB luminosity distribution in $m_{I}$ and that the difference between $\left\langle m_{\text {bol }}\right\rangle$ and $\left\langle m_{I}\right\rangle$ is the same $(0.23 \mathrm{mag})$ for the LMC and SMC carbon stars in the complete BMB sample.

The Gaussian form of the $m_{\text {bol }}$ distribution is not reduced significantly in width when the photometric IR sample alone (which has simultaneous photometry at $J$, $H$, and $K$ ) is used; the shape of Figure 9 is largely real and not a result of photographic determinations of $R$ and $I$ spread over several months for stars most of which may be slightly variable. The BMB sample is essentially complete for $\mathrm{C}$ classes $\mathrm{C}, 2-3$ and later (BMB), so the falloff of the luminosity function toward the faint end is real. Furthermore, the absence of carbon stars brighter than $m_{\text {bol }}=11.8$ in the LMC is also real; any brighter C's would have been found by the Westerlund survey, which reached only the more luninous stars but covered a much larger area than did the BMB survey.

We also note from Figure 9 that the shapes and widths of the SMC and LMC bolometric luminosity functions are quite similar. The large difference in the ratios of $C$ stars to M stars between the SMC and LMC (BMB) is not reflected in any aspect of the two luminosity distributions.

\section{b) Implications of the C Star Luminosity Function}

Based on calculations by, for example, Sackmann, Smith, and Despain (1974), Scalo, Despain, and Ulrich (1975), and Scalo (1976), most authors (for example, Richer, Olander, and Westerlund 1979) believe that luminous $\mathrm{C}$ stars are highly evolved double shell burning stars which have undergone helium shell flashes during which $\mathrm{C}$ has been dredged up from the stellar interior. Such calculations have been improved by Iben and Truran (1978, henceforth IT), who included the effects of mass loss. Renzini and Voli (1981) have further improved IT's calculation of AGB evolution and C star production by considering the effects of the initial metallicity. They have also parameterized the dredge-up efficiency, which is more consistent with the large uncertainty in the physics of this process.

Although $T_{\text {eff }}$ has been predicted as a function of luminosity and mass for double shell burning stars, we cannot derive $T_{\text {eff }}$ from broad band data for reasons discussed in $\S \mathrm{V}$. We therefore use only the luminosity distribution for the observed sample to compare with the theoretical calculations. To construct a luminosity distribution for $\mathrm{C}$ stars from the calculations of Renzini and Voli (1981, henceforth RV) requires the specification of two functions: an initial mass function, which is parameterized as a power law in initial mass, at least for $M \geq 1 M_{\odot}$, and a star formation rate, assumed to be constant with time. For illustrative purposes we assume that a star evolves at a constant rate in $\log L$ between the time it becomes a C star and when its AGB phase is terminated by complete exhaustion of the envelope through steady mass outflow or planetary nebula ejection. This assumption is not crucial since the luminosity change between the beginning and end of the $\mathrm{C}$ star phase in these models is less than 1.0 mag. In Figure 9 we show two computed bolometric luminosity functions based on the RV evolutionary tracks for low metallicity stars (their Table 1k). For these two cases initial mass functions $s=0$ and $s=2.35$ were used, where $s$ is defined by the equation

$$
\frac{d N}{d M}=M^{-s},
$$

and 2.35 is the Salpeter value. Use of the IT tracks results in a very similar luminosity function. The decline toward lower luminosities is produced by the relatively short time stars of mass close to the $\mathrm{C}$ star lower limit (approximately $2 M_{\odot}$ ) are C stars before losing their entire envelope by mass loss. The cutoff toward high luminosity is produced by the slope of the IMF (for $s>0)$ and by the upper mass limit for C stars of $8 M_{\odot}$ (IT, RV).

It is apparent from Figure 9 that a gross discrepancy exists: the observed $\left\langle M_{\text {bol }}\right\rangle$ in the SMC and LMC is -4.8 for the BMB C stars, while the predicted $\left\langle M_{\text {bol }}\right\rangle$ is brighter than -6.2 . We note that we have considered the most favorable case for the formation of low luminosity $\mathrm{C}$ stars treated by RV. A similar problem arises for the galactic $\mathrm{N}$-type carbons; with much more uncertain luminosities their $\left\langle M_{\text {bol }}\right\rangle$ is $\sim-4.8 \pm 0.5 \mathrm{mag}$ (Baumert 1974; Eggen 1972), very close to that of the extragalactic sample. It therefore seems that something is wrong in the assumptions of IT and RV regarding the details of the dredge-up process or of the mass loss processes which end AGB evolution.

Richer (1981) has also analyzed the bolometric luminosities of the Magellanic Cloud carbon stars and 
similarly suggests that $\mathrm{C}$ star formation occurs at lower core masses than in the IT or RV calculations. Scalo and Miller (1981) reach the same conclusion via analysis of the fraction of galactic $M$ giants of various spectral classes in which technetium is present from the survey of Little-Marenin and Little (1979).

Wood (1980) and Iben (1981) have used the earlier cluster C star luminosities of FPC to consider the luminosity problem, and have been able to remove part of the discrepancy between theory and observation by, in the first case, improving the convective envelope calculations, and in the second, greatly increasing the efficiency of the dredge-up process. Wood's calculations cannot readily produce stars fainter than $M_{\text {bol }}=-4.4$, while Figure 9 shows that a significant fraction of the LMC stars fall below this limit. One of Wood's conclusions is that the ages of the cluster $\mathrm{C}$ stars measured by FPC and Mould and Aaronson (1980) are younger than $1 \times 10^{9} \mathrm{yr}$, and Wood presents arguments that this age estimate is an upper limit. While we have no independent information presently on the ages of the field $\mathrm{C}$ stars, it does not seem possible that the LMC and SMC clusters containing $\mathrm{C}$ stars could be as young as $1 \times 10^{9}$ yr. Searle, Wilkinson, and Bagnuolo (1980) have presented an age ranking for a large number of Cloud clusters. With the exception of clusters NGC 152 and 2209, all of the clusters containing $C$ stars measured by FPC and by Mould and Aaronson (1980) fall in the Searle et al. groups V, VI, and VII. These age groups are certainly considerably older than $1 \times 10^{9} \mathrm{yr}$; both the photometric parameters of Searle et al. and the appearance of cluster color-magnitude diagrams give the same result, as discussed by Searle et al. Thus some of the assumptions that go into Wood's arguments must be incorrect.

Iben has carried out an extensive set of experiments designed to match the theoretical and empirical distributions, but is still left with a significant tail of highluminosity stars that are just not observed either in the BMB or WORC surveys. Such a high luminosity tail can be suppressed by a very steep IMF $(s>3)$ for stars with $M>3 M_{\odot}$ or by star formation rates significantly higher in the past than at present. The latter cannot be true for the galactic disk (Scalo and Miller 1980) and seems unreasonable for the SMC and LMC, which are believed to be perhaps somewhat younger overall than the galactic disk (Butcher 1977), given the large number of very young $O$ and $B$ supergiants in the Magellanic Clouds.

Larger mass loss rates than IT or RV assumed, the earlier formation of planetary nebulae, or some other mechanism neglected in the evolutionary tracks which removes intermediate mass stars from the AGB may also be relevant. Alternatively, Iben (1981) has suggested that such stars are missing from the BMB survey because they are hidden within optically thick circumstellar dust shells.
Two further qualitative predictions made by Iben (1981) concern the location of the peak and low luminosity edge of the bolometric luminosity distribution of AGB stars in the SMC versus the LMC. Because the SMC has a lower mean metal abundance than the LMC, the peak of the SMC distribution should be $\sim 0.2$ mag fainter and the low luminosity edge should be $\sim 0.4$ mag fainter than those of the LMC (see Iben 1981). These differences are not seen in Figure 9. Possible explanations for this disagreement could involve further details in the physics of the dredge-up process, systematic differences in the IMF's of the SMC and LMC, or perhaps even a distance modulus difference less than the 0.5 mag we and BMB have assumed.

Nevertheless, a shell flash mixing mechanism is by no means ruled out by the observed luminosity distribution of the LMC and SMC C stars. This is because the mean luminosity computed from evolutionary tracks with the assumption that one shell flash is sufficient to turn an $\mathrm{M}$ star into a C star is in fact lower $\left(\left\langle M_{\text {bol }}\right\rangle=-3.8\right.$ for $s=2.35)$ than the observed one. We await with interest detailed calculations for the new helium flash mixing mechanism suggested by Sackmann (1980), which may well be able to match the observed luminosity function of carbon stars.

\section{c) The $M$ Stars}

The large number of late $\mathrm{M}$ giants found by $\mathrm{BMB}$ in the LMC implies that most, if not all, of them must be members of that galaxy. The survey is essentially complete within the small areas photographed for giants later than M5. Since BMB do not give $R-I$ colors for most of the detected $M$ giants, we can study in detail only the subset (roughly $20 \%$ of the total) for which infrared photometry was obtained. Their mean bolometric luminosity, calculated from the data of Tables 1-3, the bolometric corrections of FPC, and for a true distance modulus of $18.6 \mathrm{mag}$, is $\left\langle M_{\text {bol }}\right\rangle=-4.86$, which is essentially equal to the mean luminosity of the BMB carbon stars of Figure 9. The dispersion about the mean for the late $\mathrm{M}$ giants is $0.56 \mathrm{mag}$, quite comparable to that of the C stars (see Table 7). BMB found $\left\langle m_{I}\right\rangle$ for their complete sample of late $\mathrm{M}$ giants to be approximately the same as that of the LMC carbon stars. If this result is sustained and extended to the detailed form of the $\mathrm{M}$ giant luminosity function, an additional important constraint can be applied to the predictions of stellar evolutionary calculations such as those of IT and $\mathrm{RV}$, which would have difficulty producing identical luminosity functions for the $\mathrm{C}$ and $\mathrm{M}$ stars (see Iben 1981). As BMB find the mean value of $m_{I}$ for their full sample of late $\mathrm{M}$ giants in the LMC to be within 0.4 mag of that of the solar neighborhood late $M$ giants, a similar situation may prevail in the galactic disk.

The LMC M giants are mostly too bright to be first ascent red giants and must be asymptotic giant branch 
stars. Given Reimers's (1975) mass loss rates, these stars must be younger and more massive than Galactic globular cluster stars in order to have survived to such high luminosities. The absence of late $M$ giants of lower luminosity implies that stars more metal-rich than but of the same age as those of 47 Tuc are not present in the LMC field. The observed late $M$ giants represent a younger metal-rich population, which is similar to that of the galactic disk and of Baade's window (Frogel 1980).

SMC-B28, the only SMC M giant with infrared data (the brightest at $I$ of the 5 found by BMB) is variable at $K$ with an amplitude of at least $0.5 \mathrm{mag}$; the large (and variable) observed $\mathrm{H}_{2} \mathrm{O}$ index is reminiscent of galactic Mira variables. Its $\mathrm{CO}$ index and amplitude of variability are too large to permit the star to be a foreground dwarf, and it is too faint to be a Mira in the galactic halo at a distance of less than $15 \mathrm{kpc}$. It seems likely that this star is a member of the SMC, with $M_{\mathrm{bol}}=-6.7$.

\section{PHYSICAL BASIS FOR THE TRENDS IN INFRARED COLORS AND INDICES}

In $\S$ III the general behavior of the infrared colors and narrow band indices of the galactic and Magellanic Cloud C stars was described. We now attempt to discern the underlying physical mechanisms which give rise to the correlations seen in Figures 1-7, and also try to explain in a qualitative manner the small differences between the galactic, LMC, and SMC samples.

\section{a) JHK Colors and Molecular Line Blanketing}

The $(J-K)_{0}$ colors of carbon stars range from 0.6 to 2.3 mag. This range in $J-K$ and in other broad band colors has usually been interpreted as a spread in effective temperature (e.g., Scalo 1976); but as Ridgway et al. (1980) have shown, color temperatures do not agree well with effective temperatures of carbon stars determined

TABLE 8

\begin{tabular}{|c|c|c|c|}
\hline C Class & $\langle J-K\rangle_{0}$ & $\sigma$ of Mean & $\begin{array}{l}\text { Number } \\
\text { of Stars }\end{array}$ \\
\hline \multicolumn{4}{|c|}{ a) Galactic Carbons (C5-C7) ${ }^{a}$} \\
\hline $\mathrm{C}, 2$. & 1.35 & 0.06 & 3 \\
\hline $\mathrm{C}, 3$. & 1.52 & 0.08 & 11 \\
\hline $\mathrm{C}, 4 \ldots \ldots \ldots$ & 1.75 & 0.14 & 14 \\
\hline C,5. & 2.01 & 0.11 & 4 \\
\hline \multicolumn{4}{|c|}{ b) WORC LMC Sample (all T classes) ${ }^{\mathrm{a}}$} \\
\hline $\mathrm{C}, 2$. & 1.04 & 0.17 & 2 \\
\hline$C, 3 \ldots \ldots \ldots$ & 1.26 & 0.07 & 7 \\
\hline$C, 4 \ldots \ldots \ldots$ & 1.28 & 0.07 & 6 \\
\hline$C, 5 \ldots \ldots$ & 1.42 & 0.06 & 7 \\
\hline
\end{tabular}

${ }^{a}$ Emission line stars excluded. directly from occultation data. This is not surprising, given the strong departures of carbon star energy distributions from those of blackbodies and the tremendous effects of molecular line blanketing (evident, for example, in Mendoza and Johnson's $1965 U$ to $L$ photometry). We believe it is more likely that the broad band colors are determined primarily by the amount of line blanketing by carbon-containing molecules, though these abundances may, in turn, be strongly coupled to the effective temperature.

To demonstrate the importance of the molecular blanketing on the infrared colors, we compute the mean $(J-K)_{0}$ color as a function of carbon abundance class for galactic carbon stars in the temperature classification C5-C7 with $K \leq 4.0$, to minimize reddening uncertainties from the sample of Table 1, Mendoza and Johnson (1965), and Frogel and Hyland (1972). ${ }^{4}$ (Small transformations were applied to ensure that all photometric data are on the system used here.) Stars with strong emission lines were omitted as they probably have extensive shells whose emission would affect the infrared colors. The results are listed in Table $8 a$, which shows that the mean $J-K$ color increases steadily as the carbon class increases. A similar result is obtained from the WORC samples of LMC carbon stars, which have $\mathrm{C}_{2}$ band strength classes, as shown in Table $8 b$. Although it has fewer stars, this sample has the advantage of more uniform reddening and internally consistent spectral classification.

To indicate that the temperature classification is not as important a parameter in predicting the infrared colors, we averaged $J-K$ colors for the same group of galactic $C$ stars with $K \leq 4.0$ and with $C$ classes 3 or 4 (i.e., $\mathrm{C}, 3$ and $\mathrm{C}, 4$ only) to produce the results shown in Table $9 a$. The WORC sample of LMC C stars was

${ }^{4}$ Although the observations of Mendoza and Johnson (1965) and Frogel and Hyland (1972) are not corrected for reddening, $K \leq 2$ for these samples, and reddening can be ignored.

TABLE 9

MEAN $J-K$ COLOR AS A Function of T Class

\begin{tabular}{cccr}
\hline \hline T Class & $\langle J-K\rangle_{0}$ & $\sigma$ of Mean & $\begin{array}{r}\text { Number } \\
\text { of Stars }\end{array}$ \\
\hline \multicolumn{4}{c}{ a) Galactic Carbons (C,3 and C,4 only) ${ }^{\mathrm{a}}$} \\
\hline $\mathrm{C} 4 \ldots \ldots \ldots$ & 1.47 & 0.07 & 4 \\
$\mathrm{C} 5 \ldots \ldots \ldots$ & 1.61 & 0.05 & 9 \\
$\mathrm{C} 6 \ldots \ldots \ldots$ & 1.54 & 0.08 & 10 \\
$\mathrm{C} 7 \ldots \ldots \ldots$ & 1.86 & 0.33 & 6 \\
\hline \multicolumn{5}{c}{$b)$ WORC C Stars of LMC (all C classes) } \\
\hline C3 $\ldots \ldots \ldots$ & 1.24 & $\ldots$ & 1 \\
C4 $\ldots \ldots \ldots$ & 1.21 & 0.07 & 11 \\
C5 $\ldots \ldots \ldots$ & 1.32 & 0.05 & 3 \\
C6 $\ldots \ldots \ldots$ & $\ldots$ & $\ldots$ & 0 \\
C7 $\ldots \ldots \ldots$ & 1.20 & $\ldots$ & 1 \\
\hline
\end{tabular}

${ }^{a}$ Emission line stars excluded. 
averaged for each temperature class over all $\mathrm{C}$ classes to yield Table $9 b$. In Tables $9 a-9 b$, there is no definite trend of color with temperature classification. A similar lack of correlation of broad band colors from $I$ to $L$ with temperature classes as determined by spectral classification was noted by Scalo (1976).

We now attempt to model in a more quantitative manner the effects of blanketing on the broad band infrared colors for a fixed effective temperature. The general effect of strong molecular line blanketing over the region between 1 and $3 \mu \mathrm{m}$ will be to smooth out the pronounced opacity minimum at $1.6 \mu \mathrm{m}$ (within the $H$ band) and to bring the colors closer to a blackbody line, as is indeed observed. This blackbody temperature will correspond to a point in the atmosphere which is relatively high, and thus cooler, reflecting the strong molecular opacity, so we expect $T_{\mathrm{BB}}(J H K)$ to be less than $T_{\text {eff }}$. This again agrees with observations, where the mean difference between $T_{\text {eff }}$ and $T_{\mathrm{BB}}(J-K)$ deduced from Walker's (1980) infrared photometry for carbon stars with occulation diameters is $400 \mathrm{~K}$. To model the effects in more detail, we have taken the calculations of absorption in the Johnson filters due to $\mathrm{C}_{2}, \mathrm{CN}$, and CO done by Price (1970) and made small corrections for the slightly different effective wavelength of our $J$ filter. These have then been applied to the colors of $M$ giants with $T_{\text {eff }}$ between 3000 and $4000 \mathrm{~K}$ (about M8 to K5) from Frogel et al. (1978) for varying amounts of $\mathrm{C}_{2}$ enhancement; the resulting colors are plotted in Figure $10{ }^{5}$ The $C_{2}$ enhancement (denoted $\left[C_{2}\right]$ ) is measured by the logarithm of the number density of $\mathrm{C}_{2}$ molecules at some mean continuum optical depth in a carbon star as compared with a solar abundance giant of the same $T_{\text {eff }}$ and surface gravity and is calculated from information given by Price. It should be noted that to produce $\left[\mathrm{C}_{2}\right]=5$ in an M0 oxygen-rich star requires an increase in the $\mathrm{C}$ abundance of less than a factor of 10 above solar, based on molecular equilibria calculations by Dolan (1965), so that the $C_{2}$ values in Figure 10 should be typical of moderate to strong carbon stars. Despite the qualitative nature of Figure 10, it does show agreement between the observed and predicted trends of the $J H K$ colors of $\mathrm{C}$ stars. We conclude that the effects of a range in the $\mathrm{C}_{2}$ abundance can produce the sequence of $J-H, H-K$ colors of galactic C stars seen in Figure 1, with the bluest $\mathrm{C}$ stars (i.e., those closest to the $\mathrm{M}$ giants) having the least $C_{2}$ enhancement, and the reddest $\mathrm{C}$ stars having the largest $\mathrm{C}_{2}$ enhancement. Strong molecular blanketing in the $1.2-3 \mu \mathrm{m}$ region makes any temperature determinations involving infrared colors particularly suspect.

The strength of the $\mathrm{C}_{2}$ blanketing for a fixed carbon abundance will also be strongly correlated with effective

\footnotetext{
${ }^{5}$ The colors computed for the higher $\mathrm{C}_{2}$ enhancement in Fig. 10 involve a slight extrapolation from Table 9 of Price.
}

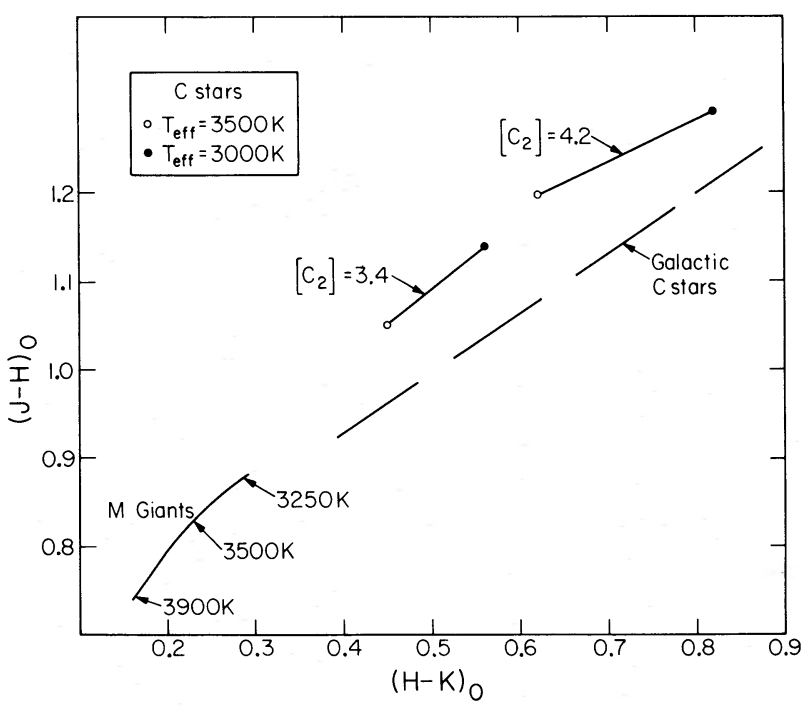

FIG. 10.- The observed mean $(J-H)_{0}$ and $(H-K)_{0}$ colors are shown for galactic $M$ giants from Frogel et al. (1978) and for galactic carbon stars from the least squares fit of Fig. 1. Approximate effective temperatures are indicated for the $M$ giants. Predicted colors for carbon stars are displayed for two effective temperatures and for two values of the $\mathrm{C}$ abundance based on Price's (1970) calculations of molecular line blanketing.

temperature. Observaions of $\mathrm{CN}$ and $\mathrm{C}_{2}$ in the galactic carbon stars (Gow 1977; Baumert 1972) can be matched by varying effective temperatures only (cf. Tsuji 1964; Johnson, Beebe, and Sneden 1975), and it is possible that the galactic $\mathrm{C}$ stars actually have a rather small range in carbon abundance (or $\mathrm{C} / \mathrm{O}$ ratios), though it is not necessary. For purposes of the subsequent discussion, we use the terms $\mathrm{C}_{2}$ abundance or $\mathrm{C}_{2}$ enhancement to describe the strength of the $\mathrm{C}_{2}$ and $\mathrm{CN}$ features (which are highly correlated in galactic C stars; see Gow 1977), even though the $\mathrm{C}$ abundance may actually be fixed and only the temperature varying.

\section{b) Narrow Band Indices}

The $\mathrm{H}_{2} \mathrm{O}$ and $\mathrm{CO}$ index measurements for carbon stars are in fact measurements of neither molecule. Instead, they measure a combination of $\mathrm{CN}, \mathrm{CO}$, and continuum slope. The two indices are defined by three filters (see Frogel et al. 1978 for details): a continuum filter centered at $2.20 \mu \mathrm{m}$, and $\mathrm{H}_{2} \mathrm{O}$ filter centered at $2.00 \mu \mathrm{m}$, and a CO filter centered at $2.36 \mu \mathrm{m}$. CO absorption affects only the $\mathrm{CO}$ filter; $\mathrm{CN}$ affects the entire $2 \mu \mathrm{m}$ region and a red continuum slope will, in the absence of other effects, produce a positive $\mathrm{H}_{2} \mathrm{O}$ index and a negative $\mathrm{CO}$ index.

Figure 3 displays the $\mathrm{CO}$ indices of galactic $\mathrm{C}$ stars as a function of $J-K$ color, and, as noted in $\S$ III, there is a strong trend, with scatter larger than the observational error, for CO to decrease as $J-K$ increases. In view of 
our statement that higher $\mathrm{C}_{2}$ abundances produce redder $J-K$ colors, this is at first sight paradoxical. However, irrespective of how large the $\mathrm{C}$ abundance may become, there can be no more $\mathrm{CO}$ in $\mathrm{C}$ stars than there are $\mathrm{O}$ atoms. In oxygen-rich stars, oxygen is approximately twice as abundant as carbon, and most of the carbon for such cool stars is tied up in $\mathrm{CO}$. Hence even an extreme $\mathrm{C}$ star will not have more than 3 times the $\mathrm{CO}$ column density (ignoring differences in the optical depth scales) of an M star of the same temperature. For $\mathrm{M}$ and late $\mathrm{K}$ giants, where the $\mathrm{CO}$ first overtone bands are saturated, an increase in CO column density by a factor of 3 will increase the observed $\mathrm{CO}$ index by less than $0.10 \mathrm{mag}$. The added effect of a red continuum will be to decrease the $\mathrm{CO}$ index with increasing $J-K$; the red continuum reduces the $\mathrm{CO}$ index by $0.15 \mathrm{mag}$ for $J-K \approx 1.5 \mathrm{mag}$ based on extrapolation of the $H-K$ colors of $\mathrm{C}$ stars. Another important factor is the opacity of $\mathrm{CN}$ throughout the $2 \mu \mathrm{m}$ region, previously suggested by Spinrad and Wing (1969), Wing and Spinrad (1970), and Faÿ et al. (1974) as the cause of abnormally weak $\mathrm{CO}$ bands in extreme $\mathrm{C}$ stars. As was discussed earlier for giants in 47 Tuc (Frogel, Persson, and Cohen 1981), there is substantially more CN line blanketing in the continuum filter bandpass than in the filter centered on the $\mathrm{CO}$ band. Thus as the $\mathrm{CN}$ bands become stronger, the flux in the continuum filter decreases and the observed $\mathrm{CO}$ index becomes smaller. Molecular equilibria calculations (e.g., Dolan 1965) indicate that $\mathrm{CN}$ in $\mathrm{C}$ stars is more than 10 times as abundant as in O-rich stars for $T<4000 \mathrm{~K}$. FTS spectra from the $4 \mathrm{~m}$ telescope at KPNO (courtesy of D. Carbon and D. Hall) for several $M$ giants and for Y CVn $(C 5,4)$ demonstrate the extreme enhancement of $2 \mu \mathrm{m} \mathrm{CN}$ absorption in $\mathrm{C}$ stars.

The sum of these three effects (more $\mathrm{CO}$, more $\mathrm{CN}$, and a redder continuum) in $\mathrm{C}$ stars as compared to $\mathrm{M}$ stars can in a qualitative manner reproduce the difference in $\mathrm{CO}$ indices between $\mathrm{C}$ and $\mathrm{M}$ stars of the same temperature and can explain the trend of smaller $C O$ indices with redder $J-K$ colors shown by the galactic $\mathrm{C}$ stars in Figure 3. A spread in the $\mathrm{O}$ abundance, which affects the amount of $\mathrm{CO}$, in the $\mathrm{N}$ abundance, which affects the $\mathrm{CN}$ absorption, in the microturbulent velocity, or in the ${ }^{12} \mathrm{C} /{ }^{13} \mathrm{C}$ ratio may give rise to the scatter in the relationship between the $\mathrm{CO}$ index and $J-K$ color. We have also ignored the possible importance of weak bands of polyatomic molecules which could coincide in wavelength with one of the relatively narrow filters used to define these indices.

Figure 5 shows that the observed $\mathrm{H}_{2} \mathrm{O}$ indices of galactic $\mathrm{C}$ stars systematically increase as $J-K$ becomes redder. This is caused entirely by changes in slope of the energy distribution on the $\mathrm{H}_{2} \mathrm{O}$ index, which is defined by a filter at $2.0 \mu \mathrm{m}$ on the $1.9 \mu \mathrm{m} \mathrm{H}_{2} \mathrm{O}$ band and a single continuum filter centered at $2.2 \mu \mathrm{m}$. The trend of
Figure 5 can be reproduced by taking the Rayleigh-Jeans approximation to a $10,000 \mathrm{~K}$ blackbody to define $\mathrm{H}_{2} \mathrm{O}$ $=0.00 \mathrm{mag}$ (for an A0 star) and then extrapolating the continuum slopes between 2.0 and $2.2 \mu \mathrm{m}$ from the broad-band photometry of $\mathrm{C}$ stars. Specifically, a line has been drawn on Figure 5 representing the $\mathrm{H}_{2} \mathrm{O}$ indices predicted from the $H-K$ colors for Mendoza and Johnson's (1965) sample of C stars (with $K \leq 4.0$ to avoid reddening effects) for $1.2 \leq(J-K) \leq 2.3$. The good agreement between this line and the distribution of Figure 5 indicates that the continuum slope alone produces the observed large $\mathrm{H}_{2} \mathrm{O}$ indices in $\mathrm{C}$ stars. ${ }^{6}$ The scatter about the mean line in Figure 2 is, as discussed in $\S$ III, consistent with observational and reddening uncertainties for the sample of Table 1.

\section{c) A Comparison Between the Galactic, LMC, and SMC Field Carbon Stars}

We now use the physical mechanism outlined in $\S \S \mathrm{V} a$ and $\mathrm{V} b$ for the galactic $\mathrm{C}$ stars to try to explain the differences in the infrared properties of the samples of field carbon stars in the three galaxies. As described in $\S$ III, a difference between the samples is seen in Figure 2, where the SMC stars tend to be bluer in $(J-H)_{0}$ by $0.05 \mathrm{mag}$ than the LMC stars for a fixed $(H-K)_{0}$ color, while the galactic $\mathrm{C}$ stars are about 0.05 mag redder in $(J-H)_{0}$ than the LMC carbon stars for a fixed $(H-K)_{0}$. The CO indices of the LMC BMB stars as shown in Figure 4 are in the mean 0.06 mag smaller for a fixed $(J-K)_{0}$ than in the galactic field stars, while the SMC sample has $\mathrm{CO}$ indices that are perhaps 0.02 mag smaller than the LMC BMB sample. In Figure 6 there is no separation in $\mathrm{H}_{2} \mathrm{O}$ index versus $J-K$ color larger than $0.02 \mathrm{mag}$ in the $\mathrm{H}_{2} \mathrm{O}$ index between the galactic, LMC and SMC samples, as is expected since the $\mathrm{H}_{2} \mathrm{O}$ index mainly measures a continuum slope.

The basic premise we shall use to attempt to explain the differences in Figures 2 and 4 is that the SMC field stars are in the mean more metal poor than those of the LMC field. Most of the carbon seen in the $C$ star atmospheres is produced via nuclear reactions which depend ultimately only on hydrogen atoms, and hence carbon abundance is independent of initial metallicity. This implies that the ratio $\mathrm{CN} / \mathrm{C}_{2}$ will increase in $\mathrm{C}$ stars as the metallicity (i.e., the $\mathrm{N}$ abundance) increases. Lloyd-Evans (1980) has in fact observed anomalously

\footnotetext{
${ }^{6}$ Although there is $\mathrm{CN}$ absorption throughout the $2 \mu \mathrm{m}$ region, the frequency dependence of the $\mathrm{CN}$ line absorption between 2.0 and $2.2 \mu \mathrm{m}$ is, for cool temperatures, sufficiently small (see Johnson, Marenin, and Price 1970) that the effect of CN absorption on the $\mathrm{H}_{2} \mathrm{O}$ index must be less than half of its effect on the $\mathrm{CO}$ index. We thus do not expect any observable scatter in the $\mathrm{H}_{2} \mathrm{O}$ index at a fixed $J-K$ to arise from reasonable $\mathrm{N}$ variations.
} 
weak $\mathrm{CN}$ bands relative to $\mathrm{C}_{2}$ bands in image tube spectra of several LMC C stars. Nearly all of the molecular band absorption at $J, H$, and $K$ in $C$ stars is due to $\mathrm{CN}$ or $\mathrm{C}_{2}$. Price's (1970) line blanketing calculations were made for solar metallicity stars and demonstrate that the ratio of the absorption of $\mathrm{CN}$ to that of $\mathrm{C}_{2}$ is larger at $J$ than at $H$ or $K$. Typical $\mathrm{CN}$ absorption at $J$ is approximately $0.1 \mathrm{mag}$ for a solar metallicity $\mathrm{C}$ star from Table 7 of Price. One therefore expects $J$ to become brighter in low-metallicity carbon stars by about $0.1 \mathrm{mag}$, but $H$ and $K$ to remain unchanged. This is the magnitude and sign of the shift in Figure 2 between the galactic, LMC, and SMC carbons, given the known mean metallicities of these three galaxies.

The case of the $\mathrm{CO}$ index is more complex. Both $\mathrm{CO}$ and $\mathrm{CN}$ (which strongly affects the flux in the continuum filter) will decrease with diminishing metallicity. Reduced CO will make the $\mathrm{CO}$ index smaller, while less $\mathrm{CN}$ will make the observed $\mathrm{CO}$ index larger. Thus it is difficult to predict how the $\mathrm{CO}$ index in carbon stars will vary with metallicity given these competing trends. A quantitative analysis requires a calculations of $\mathrm{CO}$, $\mathrm{CN}$, and $\mathrm{C}_{2}$ opacities as functions of metallicity and $\mathrm{C}$ enhancement which are at present not available.

\section{SUMMARY AND CONCLUSIONS}

Infrared photometry of a selection of the BMB complete sample of C stars in the LMC and SMC and of a sample of field $\mathrm{C}$ stars in the Galaxy shows that the basic trends of the infrared colors and indices for $\mathrm{C}$ stars in the Galaxy and the Magellanic Clouds are similar but not identical. The infrared photometry was used with the bolometric corrections of Frogel, Persson, and Cohen (1980) to derive a bolometric luminosity function for the BMB sample, which was then compared with evolutionary calculations of double shell burning stars undergoing He shell flashes by Iben and Truran (1978) and by Renzini and Voli (1981). The following are the important results:

1. The shape of the luminosity distribution in $I$ of $\mathrm{BMB}$ is essentially that of the bolometric luminosity function. Although the transformation from $I$ to $M_{\text {bol }}$ contains a color term, the difference between the mean $I$ magnitude of the SMC and LMC samples is the same as the difference between the mean bolometric magnitudes of the two samples. For distance moduli of 18.6 and 19.1 for the LMC and SMC, respectively, $\left\langle M_{\mathrm{bol}}\right\rangle$ for the $\mathrm{BMB}$ samples of $\mathrm{C}$ stars is -4.8 for both galaxies.

2. None of the available theoretical models can reproduce the SMC and LMC luminosity distribution for C stars, as $\left\langle M_{\text {bol }}\right\rangle$ is predicted to be too bright. However, the assumption that helium shell flashes are the mechanism by which dredge-up of fresh carbon transforms an oxygen-rich star into a $\mathrm{C}$ star is still tenable, if $\mathrm{M}$ stars become $\mathrm{C}$ stars at a luminosity lower than that of the presently available theoretical calculations (i.e., after fewer helium shell flashes).

3. The observed luminosity function is approximately Gaussian with a FWHM of $\sim 0.8 \mathrm{mag}$ in both the SMC and LMC. Theoretical predictions of a difference of mean luminosity and dispersion for these two galaxies are not in accord with the observed luminosity function, given a 0.5 mag difference in distance modulus between the SMC and LMC.

4. Even when compared with the new evolutionary calculations by Iben (1981), there is a scarcity of high luminosity $\left(M_{\text {bol }}<-6.0\right) \mathrm{C}$ stars observed in the Magellanic Clouds. This implies that intermediate mass stars $\left(M>3 \quad M_{\odot}\right)$ never become $\mathrm{C}$ stars in the manner described by extant calculations of AGB evolution. Some hitherto neglected physical effect must shorten the time these stars spend in the $\mathrm{C}$ star domain.

5. The infrared observations of the late $M$ giants in the BMB sample of the LMC field show that they are similar to galactic field late $\mathrm{M}$ giants in their broad band colors and indices. The limited available data suggest that the luminosity function of the late $\mathrm{M}$ giants in the LMC is essentially identical to that of the C stars.

6. The narrow band $\mathrm{H}_{2} \mathrm{O}$ and $\mathrm{CO}$ indices are strongly correlated with $J-K$ color. When the opacities of carbon-containing molecules, as calculated by Price (1970), are considered, we find that the indices measured for $\mathrm{C}$ stars behave in a way that can be predicted using the filter bandpasses, continuum slopes, and $\mathrm{CO}$ and $\mathrm{CN}$ blanketing. The observed indices do not primarily measure $\mathrm{H}_{2} \mathrm{O}$ or $\mathrm{CO}$ absorption strengths in $\mathrm{C}$ stars.

7. The small differences in the trends of colors and indices between the LMC, SMC, and galactic samples are explained by a difference in the ratio of $\mathrm{CN}$ to $\mathrm{C}_{2}$ opacity, which is a direct consequence of the relative mean metallicities of the Magellanic Clouds and the Galaxy.

8. The infrared colors of $\mathrm{C}$ stars are strongly affected by the line blanketing produced by molecules containing carbon. Thus two-color diagrams probably represent primarily sequences of increasing $\mathrm{C}_{2}$ enhancement, which most likely arises from a relatively small range in actual $\mathrm{C}$ abundances and shifts in molecular equilibria as $T$ varies. For this reason broad band colors involving the 1.2-3 $\mu \mathrm{m}$ region are not reliable temperature indicators.

We are extremely grateful to Victor Blanco, Martin McCarthy, and Betty Blanco for permitting us to use the results of their survey before publication. We also wish to acknowledge the stimulus provided by Icko Iben and Alvio Renzini.

This research was supported in part by NSF grant AST 76-22676 and by a grant from the Caltech Recycling Center. 
APPENDIX

\section{a) The Reddening Law}

In order to make a comparison between intrinsic colors of carbon stars in the three galaxies, it is necessary to correct the observed colors for reddening. Since the observed differences in the carbon star colors between the galaxies are a few hundredths of a magnitude, the corrections must be made with some care, as the average extinctions in the Galaxy, the SMC, and the LMC are not the same, nor are they small.

The general procedure which is used to obtain reddening corrections is to estimate the extinction from $U B V$ colors of early type stars near the carbon stars. The implied reddening is then applied to the various observed colors of the carbon stars. This is complicated by the large bandpasses of the filters used for most of the photometry, which cause the corrections to be applied to a star to be a function of its spectral type. Though this effect is well known (e.g., Blanco 1956), most calculations of the effect have concentrated on the $B$ and $V$ filters of the Johnson system (e.g., Blanco 1956; Honeycutt 1972; Olson 1975; but see also Dean et al. 1978; Jones and Hyland 1980). Empirical determinations of the overall reddening law have been largely restricted to early type stars (e.g., Johnson 1968; FitzGerald 1970; Schultz and Weimer 1975; Sneden et al. 1978; Dean et al. 1978) with the notable exception of the work of Lee (1970) on M supergiants. An additional complication is that work which extends to the visible necessarily involves only moderate reddening, so that coefficients in the infrared are relatively inaccurate. Work in the infrared involving large reddenings has been done (Becklin et al. 1978; Jones and Hyland 1980; see also Elias 1978), but the color excess ratios derived in these studies must be spliced on to the shorter wavelength law.

The reddening law used in this paper (Table 10) is thus a composite. The reddening law for early type stars (nominally spectral type A0) is nearly that given by Schultz and Weimer (1975), modified in the infrared using the results of Becklin et al. (1978) and Lee (1970). Shifts in the reddening law for other spectral types were computed with the analytic approximation to the Whitford (1958) law given by Miller and Mathews (1972) and by integrating various stellar energy distributions (Faÿ and Honeycutt 1972; Woolf et al. 1964; Honeycutt et al. 1977; Strecker et al. 1979; Goebel et al. 1980) over the filter response functions for the filters in Table 10 (given by Johnson 1965; Bessell 1979; and tracings of the CTIO and Las Campanas infrared filters). The shifts calculated in this way agree well with previous calculations (e.g., Olson 1975; Honeycutt 1972; and Dean et al. 1978) and with the empirical measurements by Lee (1970) for M supergiants.

The shift for the infrared filters as functions of temperature and abundance class are very small, typically 1-2\% of the reddening at the relevant wavelength, so we have adopted a mean value for all stars, as the visual extinction is less than 1 mag for all but one of the stars in this paper. For the same reason, all nonlinear terms in the reddening law have been neglected (cf. Olson 1975; Dean et al. 1978).

TABLE 10

ADOPTED REDDENING LAW

\begin{tabular}{|c|c|c|c|c|}
\hline \multirow[b]{2}{*}{$\lambda^{\mathrm{a}}$} & \multicolumn{3}{|c|}{$A_{\lambda} / X^{\mathrm{b}}$ FOR SPECTRAL TYPE } & \multirow{2}{*}{$\begin{array}{l}\text { ESTIMATED } \\
\text { UNCERTAINTY }\end{array}$} \\
\hline & $\mathrm{A} 0$ & M & $\mathrm{C}$ & \\
\hline$B$ & 1.31 & 1.22 & 1.19: & \pm 0.02 \\
\hline$\ldots$ & 1.00 & 0.96 & 0.94 & 0.01 \\
\hline$R_{\mathrm{C}}$ & 0.82 & 0.76 & 0.77 & 0.03 \\
\hline$R_{\mathrm{J}} \cdot$ & 0.75 & 0.68 & 0.69 & 0.02 \\
\hline$I_{\mathrm{C}} \ldots$ & 0.61 & 0.60 & 0.60 & 0.02 \\
\hline$I_{\mathrm{J}} \ldots$ & 0.49 & 0.46 & 0.46 & 0.02 \\
\hline$J_{\mathrm{CIT}} \cdots \ldots \ldots \ldots$ & & 0.265 & & 0.020 \\
\hline$H \ldots \ldots$ & & 0.155 & & 0.010 \\
\hline$K \ldots \ldots \ldots \ldots$ & & 0.090 & & 0.010 \\
\hline & & 0.050 & & 0.010 \\
\hline $\mathrm{CO}$ & & -0.010 & & 0.002 \\
\hline $\mathrm{H}_{2} \mathrm{O} \ldots \ldots \ldots \ldots$ & & +0.016 & & 0.003 \\
\hline$A_{V} / E_{B-V, 0} \cdots$ & 3.20 & 3.07 & 3.02 & 0.20 \\
\hline
\end{tabular}

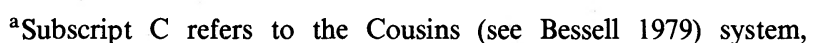
subscript J to the Johnson (1965) system, and subscript CIT to the "Caltech" (Frogel et al. 1978) system.

${ }^{\mathrm{b}} X$ is the amount of extinguishing material in the line of sight and is defined as equal to the visual extinction of a zero-color star in the limit of small reddening (see text). 
We have assumed that the reddening laws in the Magellanic Clouds do not differ significantly in the wavelength region of interest. Evidence for the LMC supports this assumption (Feast, Thackeray, and Wessselink 1960; Brück et al. 1970), while the situation in the SMC is less certain (cf. Eggen 1977; Isserstedt 1976, 1980; Madore 1977). Unless the reddening law in the SMC is grossly deviant, the results in this paper will be unaffected, as the reddening corrections are only a few hundredths (including foreground reddening).

\section{b) Individual Reddening Values}

For the galactic carbon stars (Table 6), we have used compilations of the galactic reddening distribution by FitzGerald (1968) and Lucke (1978), where the latter was given higher weight in cases of disagreement. For purposes of computing distances to the individual stars, we assumed a mean absolute $I_{C}$ of -4.8 and used the photometry of Walker (1979) to compute the apparent distance modulus. Most of the stars are at large enough $z$ distances that the uncertainty in the absolute magnitudes does not affect the derived reddening appreciably. The main source of error is in the reddening maps themselves; it seems a reasonable guess that the uncertainty in any single value for the reddening is roughly 50\%. This can be seen by examining Figure 1, where the scatter is smallest for the least-reddened stars (after correction, of course).

For the Magellanic Cloud stars the reddening was derived using early type stars in a half-degree square centered on each carbon star field; for the WORC stars we simply used the mean LMC value given by Brunet (1975). The early type star measurements were supplemented by a smaller number of measurements of $\mathrm{H}$ il regions (Dufour 1975; Dufour and Harlow 1977) and Cepheids (Martin, Warren, and Feast 1979), which did not, however, affect the derived values significantly.

For the SMC, the data for early type stars were taken from Azzopardi and Vigneau (1975) plus additional measurements by Gascoigne (1954), Feast, Thackeray, and Wesselink (1960), Wesselink (1962), and Isserstedt (1978). For the LMC, the data for early-type stars were taken from the summary by Rousseau et al. (1978), which includes most previous work, and a few measurements by Isserstedt (1979).

It should be noted that this method is subject to systematic error due to bias in the early-type star sample toward less-reddened stars. As the BMB carbon star samples are complete, their mean reddening should be larger. An approximate estimate of the size of this effect in the SMC can be made using estimates of SMC transparency from background galaxy counts (e.g., Hodge 1974; MacGillivray 1975). These suggest that the mean $A_{V}$ in the SMC fields is underestimated by at most $0.3 \mathrm{mag}$, and probably rather less. The greater degree of completeness of the LMC early star samples and examination of the dispersion in individual reddening values suggests that systematic errors in the LMC reddening estimates are comparable to the SMC errors. Any increase in the LMC and SMC reddening estimates will increase slightly the color differences already found between the galactic carbon stars and those in the Magellanic Clouds. The effects on the bolometric luminosity estimates are comparable to other sources of uncertainty.

Intrinsic colors for Magellanic Cloud early type stars were assumed to be the same as for galactic stars (Brunet 1975; Isserstedt 1976). The colors given by FitzGerald (1970) were used, supplemented by results from Brunet (1975) and Isserstedt (1976). The absolute magnitude calibration of the luminosity classes was taken from Walborn $(1972,1973)$ for types O-B2 and from Balona and Crampton (1974) for types B0 onward; average values were used in the regions of overlap.

The color excess for each early type star was computed in two ways if a spectral type was available. For the first, the procedure essentially follows Lucke (1973): the apparent magnitude and distance modulus were used to define the luminosity class for the spectral type, and the color excess was determined from the observed and intrinsic $B-V$ colors. For the second method, only the magnitudes and colors were used to match the observed $V, B-V$, and $U-B$ colors to the intrinsic values; the result is a value for the spectral type and for the reddening. For this purpose, the ratio of the color excess $E(U-B)$ to $E(B-V)$ was assumed to be 0.72 , although the ratio is in fact a function of spectral type (e.g., FitzGerald 1970). For the rather small reddening values involved, the additional iterations required did not seem to be needed.

Where values determined by the two methods agreed, they were given double weight; single weight was given to values determined from the spectral type where the photoelectric values were different by more than $0.10 \mathrm{mag}$ in $E(B-V)$. Single weight was also given to values determined by the second method where no spectral type existed.

The resulting values for the BMB fields are summarized in Table 11. A value is included for the BMB SMC wing field, though we observed no stars in this field. Our estimates for reddening are generally comparable to those computed for stars in the SMC and LMC (e.g., Tifft and Snell 1971; Brunet 1975; Azzopardi and Vigneau 1977). 
TABLE 11

EXTINCTION CORRECTION ${ }^{\mathrm{a}}$

\begin{tabular}{|c|c|c|c|}
\hline Field & $E_{B-V, 0}$ & $A_{V, 0}$ & $A_{V, \text { Carbon }}$ \\
\hline SMC Bar & 0.11 & 0.34 & 0.32 \\
\hline SMC Wing. & 0.05 & 0.15 & 0.14 \\
\hline LMC Bar West & 0.17 & 0.54 & 0.51 \\
\hline LMC Radio Center & 0.09 & 0.30 & 0.28 \\
\hline LMC Optical Center & 0.12 & 0.38 & 0.36 \\
\hline Westerlund et al. 1978 (mean) & 0.12 & 0.38 & 0.36 \\
\hline Estimated uncertainty............ & \pm 0.03 & \pm 0.10 & \pm 0.10 \\
\hline
\end{tabular}

${ }^{a}$ See text for definitions.

\section{REFERENCES}

Aaronson, M., Frogel, J. A., and Persson, S. E. 1978, Ap. J., 220, 442.

Azzopardi, M., and Vigneau, J. 1975, Astr. Ap. Suppl., 22, 285. . 1977, Astr. Ap., 56, 151.

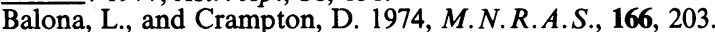

Baumert, J. 1972, unpublished dissertation, Ohio State University. 1974, Ap. J., 190, 85.

Becklin, E. E., Matthews, K., Neugebauer, G., and Willner, S. P. 1978, Ap. J., 220, 831.

Bessell, M. S. 1979, Pub. A.S.P., 91, 592.

Blanco, V. M. 1956, Ap. J., 123, 64

Blanco, B. M., Blanco, V. M., and McCarthy, M. F. 1978, Nature, 271, 638 .

Blanco, V. M., McCarthy, M. F., and Blanco, B. M. 1980, Ap. J., 242, 938 (BMB).

Brück, M. T., Lawrence, L. C., Nandy, K. N., Thackeray, A. D., and Wood, R. 1970, Nature, 225, 531.

Brunet, J. P. 1975, Astr. Ap., 43, 345.

Butcher, H. 1977, Ap. J., 216, 372.

Dean, J. F., Warren, P. R., and Cousins, A. W. J. 1978, M.N.R.A.S., $183,569$.

Dolan, J. F. 1965, Ap. J., 142, 1621

Dufour, R. J. 1975, Ap. J., 195, 315

Dufour, R. J., and Harlow, W. V. 1977, Ap. J., 216, 706.

Eggen, O. J. 1972, Ap. J., 174, 45 1977, Ap. J. Suppl., 34, 1

Elias, J. H. 1978, Ap. J., 224, 857.

Faÿ, T., and Honeycutt, R. K. 1972, A.J., 77, 29

Faÿ, T. D., Jr., Warren, W. H., Jr., Johnson, H. R., and Honeycutt, R. K. 1974, A.J., 79, 634 .

Feast, M. W., Thackeray, A.D., and Wesselink, A. J. 1960, M.N.R.A.S., 121, 337

FitzGerald, M. P. 1968, A.J., 73, 983. 1970, Astr. Ap., 4, 234.

Frogel, J. A. 1980, Erice Workshop on Physical Processes in Red Giants (Dordrecht: Reidel), in press.

Frogel, J. A., Cohen, J. G., Persson, S. E., and Elias, J. H. 1980, Erice Workshop on Physcial Processes in Red Giants (Dordrecht: Reidel), in press.

Frogel, J. A., and Hyland, A. R. 1972, Proc. 17th International Astrophysics Symposium, Liège, p. 111.

Frogel, J. A., Persson, S. E., Aaronson, M., and Matthews, K. 1978, Ap.J., 220, 75 .

Frogel, J. A., Persson, S. E., and Cohen, J. G. 1980, Ap. J., 239, 495 (FPC). 1981, Ap. J., 246, 842

Gascoigne, S. C. B. 1954, Australian J. Phys. Ap. Suppl., 17, 23.

Goebel, J. H., et al. 1980, Ap. J., 235, 104.

Gow, C. E. 1977, Pub. A.S.P., 89, 510.

Hodge, P. W. 1974, Ap. J., 192, 21.

Honeycutt, R. K. 1972, Pub. A.S.P., 84, 823.

Honeycutt, R. K., Ramsey, L. W., Warren, W. H., Jr., and Ridgway, S. T. 1977, Ap.J., 215, 584.

Iben, I., Jr. 1981, $A p$. J., 246, 278.

Iben, I., Jr., and Truran, J. W. 1978, Ap. J., 220, 980 (IT)

Isserstedt, J. 1976, Astr. Ap., 47, 463. 1978, Astr. Ap. Suppl., 33, 193. 1979, Astr. Ap. Suppl., 38, 239.
Isserstedt, J. 1980, Astr. Ap., 83, 322.

Johnson, H. L. 1965, Ap. J., 141, 923. 1968, in Nebulae and Interstellar Matter, ed. B. M. Middlehurst and L. H. Aller (Chicago: University of Chicago Press), p. 167

Johnson, H. R. 1978, Ap. J., 223, 238

Johnson, H. R., Beebe, R. F., and Sneden, C. 1975, Ap. J. Suppl., 29, 123.

Johnson, H. R., Marenin, I. R., and Price, S. D. 1970, in Proc. Conference Late Type Stars, ed. G. W. Lockwood and H. M. Dyck (KPNO Contribution 554)

Jones, T. J., and Hyland, A. R. 1980, M.N.R.A.S., 192, 359

Kukarkin, B. V., et al. 1969, General Catalog of Variable Stars (Moscow: Moscow University Press).

Lee, T.A. 1970, Ap. J., 162, 217.

Little-Marenin, I. R., and Little, S. J. 1979, A.J., 84, 1374.

Lloyd-Evans, T. 1980, M.N.R.A.S., 193, 97.

Lucke, P. B. 1973, Ph.D. thesis, University of Washington. 1978, Astr. Ap., 64, 367.

MacGillvray, H. T. 1975, M.N.R.A.S., 170, 241.

Madore, B. F. 1977, Astr. Ap., 55, 135.

Martin, W. L., Warren, P. R., and Feast, M. W. 1979, M.N.R.A.S., 188, 139.

Mendoza, E. E., and Johnson, H. L. 1965, Ap. J., 141, 161

Miller, J. S., and Mathews, W. G. 1972, Ap. J., 172, 593.

Mould, J., and Aaronson, M. 1980, Ap. J., 240, 464

Olson, B. I. 1975, Pub. A.S.P., 87, 349

Osmer, P. S. 1976, Ap. J., 203, 352.

Peimbert, M., and Torres-Peimbert, S. 1976, Ap. J., 203, 581.

Price, S. D. 1970, Ph.D. thesis, Ohio State University.

Reimers, S. D. 1975, in Problems in Stellar Atmospheres and Envelopes, ed. B. Bascheck, W. H. Kegel, and G. Traving (Berlin: Springer-Verlag), p. 229.

Renzini, A., and Voli, M. 1981, Astr. Ap., 94, 175 (RV)

Richer, H. B. 1981, Ap. J., 243, 744.

Richer, H. B., Olander, N., and Westerlund, B. E. 1979, Ap. J., 230, 724.

Ridgway, S. T., Jacoby, G. H., Joyce, R. R., and Wells, D. C. 1980, A.J., 85, 1496.

Rousseau, J., Martin, N., Prévot, L., Rebeirot, E., Robin, A., and Brunet, J. P. 1978, Astr. Ap. Suppl., 31, 243.

Sackmann, I.-J. 1980, Ap. J. (Letters), 241, L37.

Sackmann, I.-J., Smith, R. L., and Despain, K. H. 1974, Ap. J. 187, 555.

Sanduleak, N., and Philip, A. G. D. 1977, Pub. Warner and Swasey Obs., Vol. 2, No. 5 .

Scalo, J. M. 1976, Ap. J., 206, 474

Scalo, J. M., Despain, K. H., and Ulrich, R. K. 1975, Ap. J., 196, 805

Scalo, J. M., and Miller, G. E. 1980, Ap. J., 239, 953. 1981, Ap. J., 246, 251.

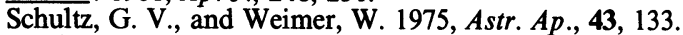

Searle, L., Wilkinson, A., and Bagnuolo, W. G. 1980, Ap. J., 239, 803.

Sneden, C., Gehrz, R. D., Hackwell, J. A., York, D. G., and Snow, T. 1978, Ap. J., 223, 168.

Spinrad, H., and Wing, R. F. 1969, Ann. Rev.Astr. Ap., 7, 249. 
Stephenson, C. B. 1973, A General Catalog of Cool Carbon Stars (Publications of Warner and Swasey Observatory, No. 4).

Strecker, D. W., Erickson, E. F., and Witteborn, F. C. 1979, Ap. J. Suppl., 41, 501 .

Tifft, W. G., and Snell, C. M. 1971, M.N.R.A.S., 151, 365.

Tsuju, T. 1964, Pub. Ann. Tokyo Obs., 9, 1.

Walborn, N. R. 1972, A.J., 78, 1067 1973, A.J., 77, 312.

Walker, A. R. 1979, South African Astr. Obs. Circ., Vol. 1, No. 4, p. 112.

. 1980, M.N.R.A.S., 190, 543

Wesselink, A. J. 1962, M.N.R.A.S., 124, 359
Westerlund, B. E 1960, Uppsala Ann., 4, 7. 1964, in The Galaxy and the Magellanic Clouds, IAU-URSI Symp. No. 20, ed. F. J. Kerr and A. W. Rodgers (Canberra: Australian Academy of Sciences), p. 239.

Westerlund, B. E., Olander, N., Richer, H. B., and Crabtree, D. R. 1978, Astr. Ap. Suppl., 31, 61 (WORC).

Whitford, A. E. 1958, A.J., 63, 201

Wing, R. F., and Spinrad, H. 1970, Ap. J., 159, 973.

Wood, P. R. 1980, Erice Workshop on Physical Processes in Red Giants (Dordrecht: Reidel), in press.

Woolf, N. J., Schwarzchild, M., and Rose, W. K. 1964, Ap. J., 140, 833.

J. G. CohEn: Dept. of Astronomy, California Institute of Technology, 105-24, Pasadena, CA 91125

J. H. Elias and J. A. Frogel: Cerro Tololo Inter-American Observatory, Casilla 603, La Serena, Chile.

S. E. Persson: Mount Wilson and Las Campanas Observatory, 813 Santa Barbara St., Pasadena, CA 91101 